\title{
Aproximación al uso y aprovechamiento de insectos comestibles en las co- munidades indígenas del oriente amazónico colombiano
}

\author{
Approach to the use of edible insects by indigenous communities of the eastern Colombian \\ amazon
}

\author{
Héctor Jaime Gasca-Álvarez*1,3,4 \\ https://orcid.org/0000-0002-0070-0715 \\ scarab7@gmail.com
William González ${ }^{2}$
https://orcid.org/0000-0003-3307-2345
willigon12@yahoo.es

\section{*Corresponding author}

1 Instituto Amazónico de Investigaciones Científicas SINCHI, Sede de Enlace, Calle $20 N^{\circ}$ 5-44, Bogotá, Colombia.

2 Instituto Amazónico de Investigaciones Científicas SINCHI, Subsede Mitú, Carrera 14 \# 10 - 37, Centro, Mitú, Vaupés, Colombia.

3 Programa de Biología, Universidad Pedagógica y Tecnológica de Colombia, Avenida Central del Norte 39-115, Sede Central - Tunja, Boyacá, Colombia.

4 Programa de Investigación, Corporación Sentido Natural, Bogotá, Colombia.

\section{Citación}

Gasca-Álvarez HJ, González W. 2021. Aproximación al uso y aprovechamiento de insectos comestibles en las comunidades indígenas del oriente amazónico colombiano. Revista peruana de biología 28(4): e21227 (Noviembre 2021). doi: http://dx.doi.org/10.15381/ rpb.v28i4.21227

$\begin{array}{ll}\text { Presentado: } & 14 / 09 / 2020 \\ \text { Aceptado: } & 15 / 10 / 2021 \\ \text { Publicado online: } & 26 / 11 / 2021 \\ \text { Editor: } & \text { Diana Silva }\end{array}$

\begin{abstract}
Resumen
En la Amazonía colombiana, los insectos constituyen un recurso natural abundante, el cual es aprovechado mediante el consumo de especies comestibles. Históricamente, los pueblos indígenas son entomofágicos y han incluido a insectos y otros artrópodos en sus dietas. En el presente estudio, se analiza el uso tradicional de insectos comestibles en las comunidades del oriente amazónico de Colombia. Durante el período de agosto 2020 a marzo 2021, se realizaron entrevistas abiertas y semiestructuradas a habitantes del municipio de Mitú (Vaupés) y de 41 comunidades indígenas aledañas, las cuales indagaron sobre la percepción, aprovechamiento y comercialización de insectos comestibles. De acuerdo con su valor de uso, Rhynchophorus palmarum (Coleoptera: Curculionidae), Syntermes spinosus (Blattodea: Termitidae), Atta laevigata (Hymenoptera: Formicidae), Lusura chera (Lepidoptera: Notodontidae) y Polybia liliacea (Hymenoptera: Vespidae), constituyen las especies de insectos comestibles más representativas de la región. Para cada especie se estableció su calendario ecológico y se documentaron sus técnicas y métodos de recolecta, sus formas tradicionales de preparación y consumo, y sus actividades de comercialización. Se resalta el potencial de las especies de insectos comestibles analizadas para el fortalecimiento de estrategias de uso sostenible direccionadas a mejorar la seguridad alimentaria de la población y el mantenimiento de los conocimientos tradicionales de las comunidades.
\end{abstract}

\section{Abstract}

In the Colombian Amazon, insects are an abundant natural resource, and part of the use of this resource is the consumption of some edible species. Historically, indigenous peoples are entomophagic and have included insects and other arthropods in their diets. In this paper, the traditional use of edible insects in the communities of the eastern Amazon of Colombia is analyzed. During August 2020 to March 2021, opened and semi-structured interviews carried out with inhabitants of the municipality of Mitú (Vaupés) and 41 surrounding indigenous communities were applied, inquiring about the perception, use and commercialization of edible insects. According to its use value, Rhynchophorus palmarum (Coleoptera: Curculionidae), Syntermes spinosus (Blattodea: Termitidae), Atta laevigata (Hymenoptera: Formicidae), Lusura chera (Lepidoptera: Notodontidae), and Polybia liliacea (Hymenoptera: Vespidae), are the most representative species of edible insects in the region. For each species, its ecological calendar was established, and its collecting techniques and methods, traditional forms of preparation and consumption, and marketing activities were documented. The potential of the edible insect species documented is highlighted to promote sustainable use strategies, with the aim of improving the food security of the population and maintaining the traditional knowledge of these communities.

Palabras clave:

Etnoentomología; entomofagia; insectos comestibles; seguridad alimentaria; antropoentomofagia; conocimiento tradicional; mojojoy; manivara; tapurú.

Keywords:

Ethnoentomology; entomophagy; edible insects; food safety; anthropoentomophagy; traditional knowledge; mojojoy; manivara; tapuru. 


\section{Introducción}

Los insectos comestibles constituyen un recurso alimenticio natural renovable y son consumidos como suplemento nutritivo o como constituyente principal de la dieta de diferentes pueblos en muchas regiones del mundo (Costa-Neto 2003, Mitsuhashi 2017, Halloran et al. 2018, Mariod 2020, Ponce-Reyes \& Lessard 2021). La entomofagia es una práctica históricamente antigua y ampliamente diseminada, estando presente en más de 3000 grupos étnicos en cerca de 130 países en el mundo (Ramos-Elorduy 2009, Tobolkova 2019). Actualmente, se ha sugerido que el consumo de insectos como alimento podría surgir en algunos casos, como una actividad sostenible, proporcionando beneficios nutricionales, ecológicos y económicos para las comunidades rurales. Sin embargo, el consumo de insectos es poco frecuente, debido a que la mayoría de los seres humanos, en especial los habitantes de las grandes ciudades del mundo consideran que alimentarse de insectos es una práctica "primitiva", ya que, por razones estéticas y sicológicas, la mayoría de los insectos son considerados animales nocivos, sucios, transmisores de enfermedades y vistos como plagas (Costa-Neto 2003). Sumado a esto, el aumento de la producción de alimentos modernos, el cambio de estructuras sociales y los cambios en la demografía en algunas regiones del mundo, hacen que el consumo de insectos no sea una opción en la alimentación diaria (Yen 2010). Sin embargo, esta idea ha cambiado a lo largo de los años, y muchos países europeos y asiáticos, incluso en América Latina, se han abierto a esta nueva perspectiva donde los insectos son buscados y consumidos por millones de personas (Ramos-Elorduy 2009, Skotnicka et al. 2021).

Los insectos han sido reconocidos en todo el mundo como un alimento nutritivo ya que proporcionan proteínas (aminoácidos como metionina, cisteína, lisina y threonina), carbohidratos, grasas, algunos minerales y vitaminas, brindando alto valor energético (Johnson 2010, Xiaoming et al. 2010, Pino-Moreno \& Ganguly 2016, Kim et al. 2019). Los altos contenidos proteicos encontrados en los insectos, inclusive con valores más altos como los contenidos en la carne de res o de pollo, es una indicación de que los insectos comestibles pueden ser de valor nutricional y eventualmente podrían reemplazar mayor proteína animal generalmente ausente en la dieta de los habitantes rurales en los países en desarrollo (Banjo et al. 2006, Guiné et al. 2021). De esta manera, la entomofagia puede ser considerada como una solución práctica y viable para la seguridad alimentaria, teniendo en cuenta el aumento de las poblaciones, el incremento del consumo y la posible disminución de la disponibilidad de alimentos (Gahukar 2011, Seni, 2017, Selaledi et al. 2021). Se estima que alrededor de 2100 especies de insectos son consumidas en el mundo, especialmente en países de Asia, África y de Latinoamérica (Ramos-Elorduy 2009, van Huis et al. 2013, Jongema 2017, Tobolkova 2019). De estas especies, se pueden consumir los diferentes estados de su desarrollo. De algunas se consumen los huevos, de otras las larvas y las pupas, y de otras solamente los adultos. Entre los órdenes de insectos comestibles más importantes se encuentran Coleoptera, Hymenoptera, Blattodea, Lepidoptera, Odonata y Orthoptera. Coleoptera es uno de los órdenes con los contenidos más altos de proteína bruta, dentro del cual se han reportado cerca de 700 especies comestibles, lo que corresponde aproximadamente al 33\% de la estimación global (van Huis et al. 2013, Bernard \& Womeni 2017, Jongema 2017).

El estudio de la percepción, conocimiento y uso de los insectos por parte de las culturas humanas permite reconocer diferentes modelos para el manejo de la biodiversidad y abordar temas como la educación biocultural y el establecimiento de bases socioculturales para la conservación y uso sostenible de la biodiversidad (Costa-Neto et al. 2012, Baiano 2020). El conocimiento del potencial de los insectos comestibles es un campo de estudio que debe ser investigado y difundido entre los consumidores, especialmente en los grupos indígenas y rurales. Esta información puede complementarse mediante la documentación de los modos de vida de las comunidades, dependiendo de los conocimientos tradicionales y del uso de los insectos como fuente de alimento. Así mismo, el conocimiento tradicional ecológico (etnoecología) puede proporcionar una oportunidad para la implementación de programas de conservación de insectos y, en última instancia, en la seguridad alimentaria de las poblaciones (DeFoliart 2005, Gahukar 2011, Van Huis 2013, Baiano 2020, Guiné et al. 2021).

En Colombia, el uso de insectos comestibles ha sido poco estudiado. A manera de revisión, Arango-Gutiérrez (2011) proporciona una reseña histórica sobre la entomofagia en Colombia, en donde los casos más comunes están relacionados con el consumo de hormigas y miel de abejas melíferas. Molina (2015) realiza una breve exploración sobre los insectos consumidos en el país, reconociendo aproximadamente 48 especies de diferentes órdenes de insectos, empleadas como alimento. Actualmente se conocen cerca de 69 especies de insectos comestibles en Colombia (Gasca-Álvarez \& Costa-Neto, en preparación). Se conocen algunos estudios sobre el uso tradicional de los insectos en comunidades indígenas amazónicas. Para los pueblos Andoke del medio Caquetá, se ha documentado la simbología de las abejas sin aguijón (Jara 1996) y las mariposas (Gómez 2003). Cabrera y Nates (1999) estudiaron el uso tradicional e importancia de las abejas sin aguijón por parte de los indígenas Nukak, mientras que el conocimiento etnoentomológico de los Uitoto sobre los escarabajos fue estudiado por Gasca-Álvarez (2005). En cuanto a la entomofagía, es bien conocido el consumo de las larvas del escarabajo picudo Rhyncophorus palmarum (L.) (Coleoptera: Curculionidae) (Dufour 1987, Cerda et al. 1999, Cristancho-Sánchez \& Barragán-Fonseca 2011). Esta especie es una de las principales fuentes de alimento animal para numerosas comunidades indígenas de la Amazonía colombiana, y su uso ha sido analizado con el objetivo de proponer este recurso natural como una alternativa sostenible para la producción de proteína animal (Cristancho-Sánchez \& Barragán-Fonseca 2011). En la comunidad indígena Murui de San José del Encanto, ubicada en el noroeste de la Amazonía, cerca del departamento de Putumayo y 
la frontera colombo-peruana, se han registrado más de 30 grupos de insectos comestibles utilizados también en rituales (Reyes-Mora 2011). Osorno-Muñoz et al. (2014) evaluaron la fauna de consumo de las etnias Tuyuca y Bará en las comunidades de Bella Vista de Abiyú y Puerto Loro de Macucú, al suroriente del municipio de Mitú, en el departamento del Vaupés, registrando 28 especies de insectos comestibles incluidos en la dieta de alimentación de la población.

La diversidad de insectos comestibles en Colombia, y en particular en la Amazonía, está subestimada. Estudios etnobiológicos pueden proporcionar información para el conocimiento de su biodiversidad en el territorio amazónico colombiano, identificando especies de interés para su aprovechamiento y conservación. Teniendo en cuenta la necesidad de generar información sobre el uso de insectos comestibles por parte de las comunidades indígenas de Colombia, la presente investigación tiene como objetivo contribuir al conocimiento, valoración y aprovechamiento de los recursos naturales en la Amazonía colombiana, analizando la diversidad, uso, aprovechamiento y comercialización de las principales especies de insectos empleados como alimento en las comunidades indígenas del oriente amazónico de Colombia. De esta manera, es posible obtener información preliminar que permita entender cómo este aprovechamiento cultural puede enmarcarse en iniciativas de sostenibilidad biológica y seguridad alimentaria de las comunidades.

\section{Material y métodos}

Área de estudio. - La investigación se desarrolló con la colaboración y participación de los habitantes del municipio de Mitú, capital del departamento del Vaupés $\left(01^{\circ} 07^{\prime} 47.30^{\prime \prime} \mathrm{N}-70^{\circ} 0^{\prime} 16.35^{\prime \prime} \mathrm{W}\right)$; y en comunidades aledañas a Mitú (ver anexo 4). Varias de las comunidades se encuentran a orillas del río Cuduyarí, y dentro del área correspondiente al territorio de la etnia Cubeo (Fig. 1).
Mecanismo de investigación etnoentomológica. En el trabajo con cada una de las comunidades se identificaron y seleccionaron a las personas que participaron en la investigación, de acuerdo con las siguientes especificaciones: sexo, edad, etnia o grupo indígena, comunidad donde habita y oficio si aplica.

La información obtenida sobre insectos comestibles fue recopilada por medio de entrevistas abiertas (conversaciones casuales) y semi-estructuradas (lista de temas o preguntas que se desean cubrir), realizadas durante el período agosto 2020 - marzo 2021. En el evento de respuestas en lengua local, se contó con traducción al español. Se emplearon cuestionarios para la identificación del potencial de los insectos comestibles (Anexo 1 y 2), los cuales recopilaron la siguiente información:

a) Percepción de los insectos, insectos más comunes, insectos usados como alimento.

b) Calendario ecológico de especies comestibles (época y hora adecuadas para recolectar, tiempo empleado en la recolecta, conocimiento tradicional sobre ecología y hábitats).

c) Técnicas de captura y métodos tradicionales para encontrar, recolectar y disponer los especímenes antes de su preparación como alimento y,

d) Modo tradicional de preparación del alimento, tiempos de cocción, acompañamientos, entre otros aspectos.

Las entrevistas individuales tuvieron una duración media de 30 minutos. Los objetivos de la investigación fueron previamente explicados de manera clara a cada persona al inicio de la entrevista, con el objetivo de contar con el consentimiento de los entrevistados (Fig. 2).

Para evaluar la comercialización de insectos comestibles, se realizaron entrevistas con comerciantes de la

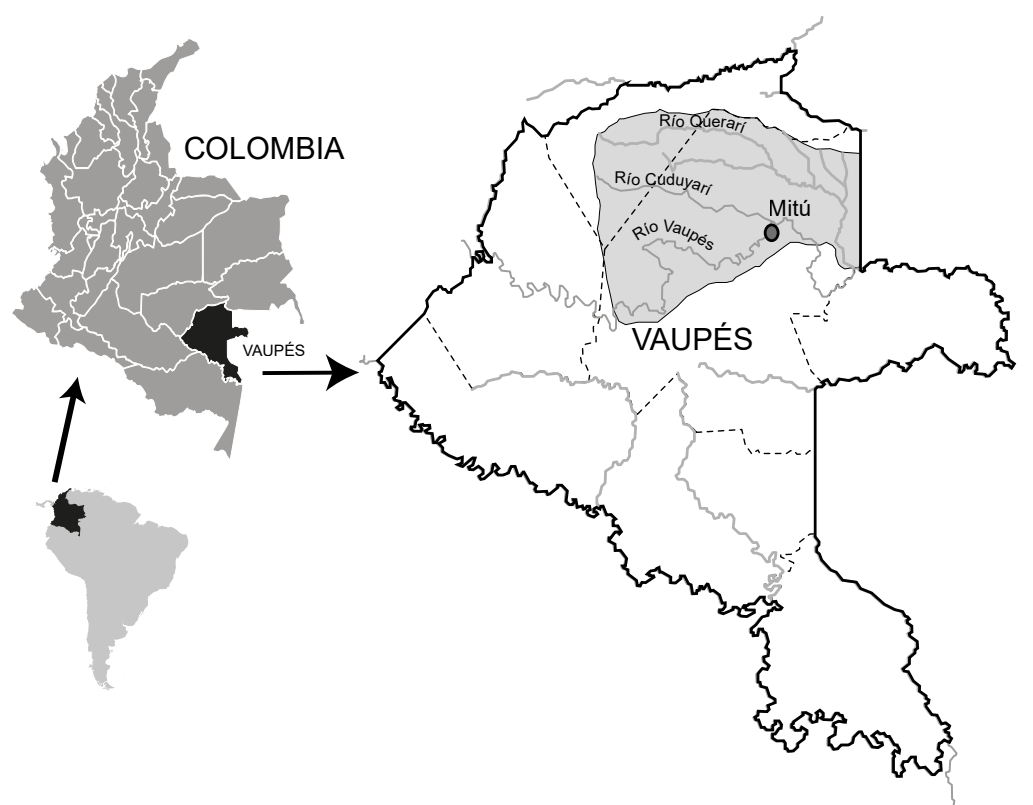

Figura 1. Ubicación del área del estudio y localización del pueblo Cubeo (en gris) en el Vaupés, Colombia. (Basado en: Corporación C.D.A 2013). 
plaza de mercado de San Victorino en el municipio de Mitú (Anexo 3). Se registró información sobre las características de comercialización, con base en los siguientes indicadores: (a) formas de comercialización; (b) procedencia del producto; (c) precio compra/venta; (d) calidad del producto; (e) factores que afectan la comercialización del producto.

Las especies de insectos comestibles se determinaron mediante la consulta de trabajos taxonómicos (Constantino 1995, Becker 2014, Fernández et al. 2015) y acudiendo a expertos para su confirmación. Los insectos en estado adulto fueron preservados en alcohol al 70\%, los especímenes en estado larval fueron preservados en solución Pampel. El material colectado fue catalogado, debidamente etiquetado y depositado en la colección de entomología del Instituto de Ciencias Naturales (Universidad Nacional de Colombia).

Análisis de datos.- Se realizó un análisis cuantitativo para calcular el valor de uso de cada una de las especies comestibles, mediante la fórmula:

$$
U V=U / n
$$
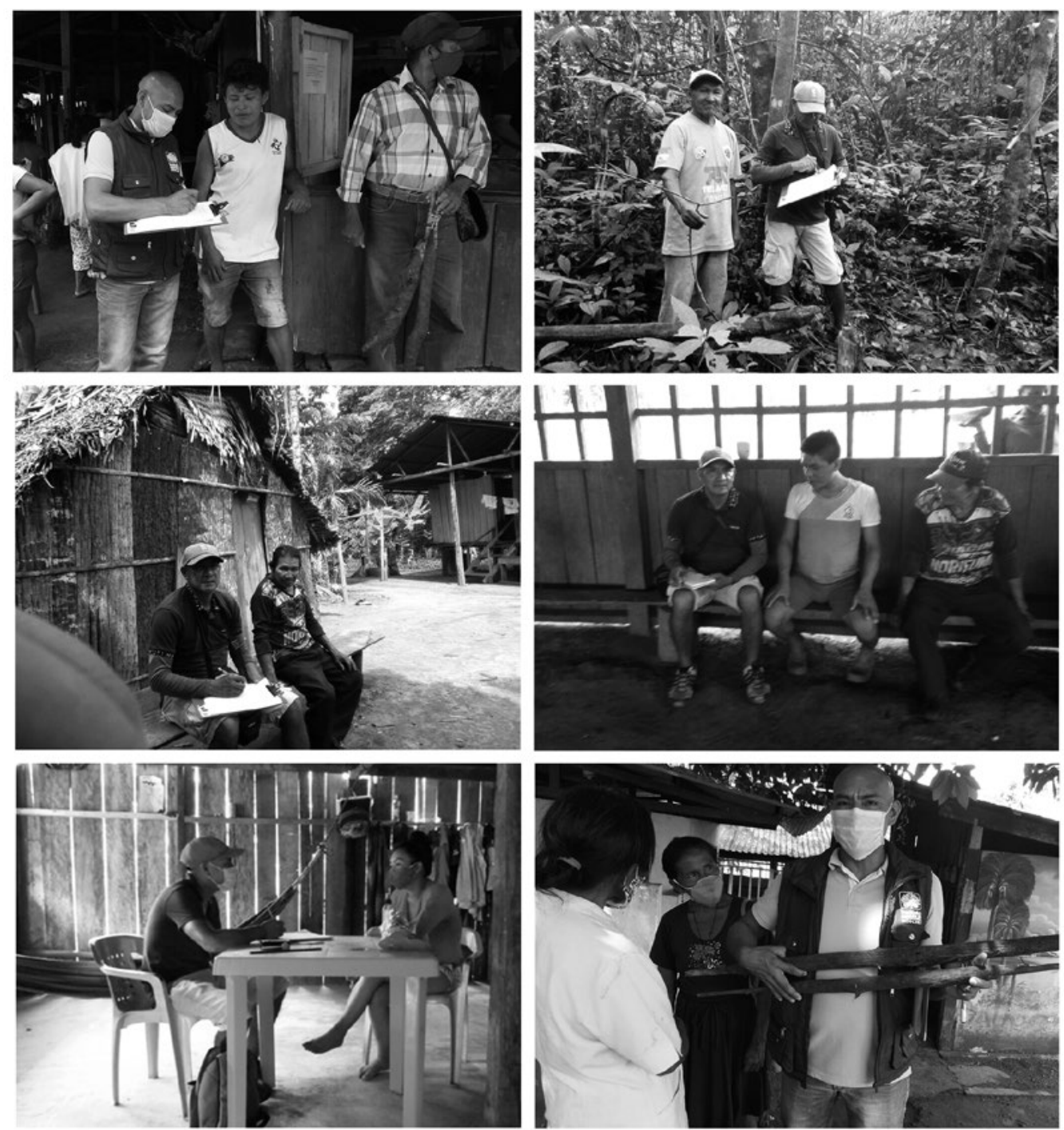

Figura 2. Entrevistas realizadas a pobladores de diferentes comunidades indígenas del oriente amazónico.

Figura 2. Entrevistas realizadas a pobladores de diferentes comunidades indigenas del oriente amazónico.
Donde $U V$ es el valor de uso de la especie, $U$ es el número de menciones por especie y $n$ es el número total de entrevistados (Phillips et al. 1994, Mozhui et al. 2017). Para cada especie comestible, se calculó un índice de valor de uso $U V_{1}$ relacionado con la percepción de los insectos como un recurso alimenticio, y un índice de valor de uso $U V_{2}$ asociado a la importancia que tiene la especie de insecto comestible dentro de la cultura de las comunidades.

\section{Resultados}

Se realizaron 137 entrevistas en las que participaron 133 personas habitantes del casco urbano del municipio de Mitú y de 41 diferentes comunidades aledañas (Anexo 4). Fueron entrevistadas 73 mujeres y 60 hombres que se encontraban en un rango de edad de 13 a 71 años (Tabla 1). De la muestra total de entrevistados, 35 personas respondieron un cuestionario sobre la percepción que tienen de los insectos en su comunidad; 41 personas contestaron preguntas relacionadas con el uso de insectos como fuente de alimento; y 61 personas proporcionaron información referente a la comercialización de insectos comestibles (Tabla 2). Los entrevistados pertenecen 
a 12 grupos indígenas o etnias: Bará, Carapana, Cubeo, Desano, Guananao, Macuna, Piratapuya, Siriana, Tatuya, Tucano y Yuruti, Waniba (Fig. 3).

Tabla 1. Características demográficas en el área de estudio.

\begin{tabular}{cl}
\hline Variables Sociodemográficas & Número de informantes \\
\hline Genero & \\
\hline Mujeres & $73(54.9 \%)$ \\
Hombres & $60(45.1 \%)$ \\
Grupo de edad & \\
\hline $13-22$ & $11(8.2 \%)$ \\
$23-32$ & $33(23.8 \%)$ \\
$33-42$ & $24(18 \%)$ \\
$43-52$ & $36(27 \%)$ \\
$53-62$ & $18(13.5 \%)$ \\
$63-72$ & $11(8.2 \%)$ \\
\hline
\end{tabular}

Tabla 2. Tipo de cuestionarios (entrevistas) realizados a los habitantes de las comunidades del oriente amazónico (Mitú, Vaupés) $\mathrm{N}=$ personas entrevistadas.

\begin{tabular}{lll}
\hline Tipo de cuestinonario & $\mathbf{N}$ & Información recopilada \\
\hline Percepción de insectos & 35 & $\begin{array}{l}\text { Cómo percibe los insectos, son } \\
\text { benéficos o perjudiciales, qué } \\
\text { insectos conoce? }\end{array}$ \\
$\begin{array}{lll}\text { Uso y aprovechamiento } \\
\text { de insectos comestibles }\end{array}$ & 41 & $\begin{array}{l}\text { Cuales insectos son comestibles, } \\
\text { dónde los recolecta, en que épo- } \\
\text { ca los recolecta, cuanto tiempo } \\
\text { emplea, como prepara los insec- } \\
\text { tos para el consumo? }\end{array}$ \\
$\begin{array}{lll}\text { Comercialización de } \\
\text { insectos comestibles }\end{array}$ & 61 & $\begin{array}{l}\text { Cómo comercializa los insectos, } \\
\text { dónde, con qué frecuencia, } \\
\text { cómo obtiene el producto, precio } \\
\text { y calidad del producto, factores } \\
\text { que afectan la comercialización }\end{array}$ \\
\hline Total entrevistas & 137 & \\
\hline
\end{tabular}

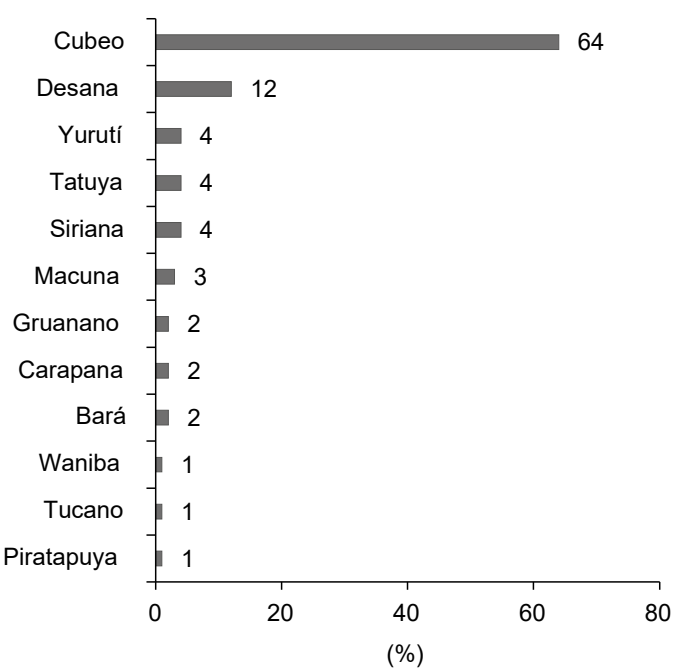

Figura 3. Porcentaje de participación de las etnias indígenas involucradas en la investigación.
Percepción de los insectos en las comunidades indígenas del oriente amazónico. - Para las comunidades indígenas del oriente amazónico colombiano, los insectos son considerados como "animalitos pequeños", "chiquiticos", "todos los chiquititos que caminan", "bichos pequeños" o "bichos raros", que se encuentran en la naturaleza, en la selva, en la chagra, en el monte o en el patio de la casa, que son parte del control del medio ambiente, "bichos de la naturaleza" y que algunos pueden ser usados como alimento. Se pueden encontrar definiciones por parte de los Desano como esta: - "Algo que no tiene sangre pero que es un animalito viviente". Se refieren aquí, a organismos de diferentes grupos de artrópodos.

Los "insectos" más comunes, conocidos por las comunidades, son hormigas, moscas, avispas, cucarrones, abejas, comejenes, mariposas, grillos, saltamontes, libélulas, tábanos, arañas, chicharras, ciempiés, zancudos, orugas y gusanos (larvas de coleópteros) de diferente tipo como el gusano del guamo, el gusano del guayaco y el gusano de bamba. Algunos insectos son comestibles y otros no. Es interesante encontrar que, para algunas personas entrevistadas, las lombrices y los caracoles son considerados en su percepción como "insectos". Los lugares más frecuentes para buscar y encontrar insectos son los bosques primarios, el monte bravo, rastrojos, chagras, pastizales, la selva, el patio de la casa, a la orilla del río, rebalses, y más específicamente en hábitats cómo troncos podridos, el suelo, en las hojas de los árboles y a orillas de caños y ríos.

De manera general, existe una opinión dividida sobre la percepción de los insectos en cuanto a su función como animales beneficiosos o perjudiciales. La mitad de los entrevistados consideran que los insectos son dañinos. Las hormigas arrieras son perjudiciales para los cultivos de las chagras; las moscas, tábanos y zancudos pican y chupan la sangre de la persona produciendo enfermedades y alergias a niños y adultos; las avispas pican, y en general, si a los insectos se les molesta atacan como un mecanismo de defensa. El 21\% de la población entrevistada considera que los insectos son inofensivos, beneficiosos o "buenos", ya que muchos de ellos sirven como alimento y si no se les molesta "no hacen nada", mientras que el 29\% opinan que algunos insectos son dañinos mientras que otros son inofensivos y sirven como alimento (Fig. 4).

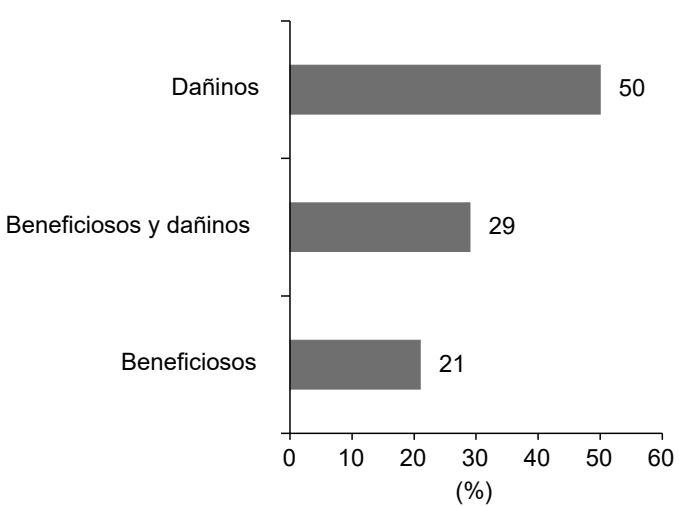

Figura 4. Percepción de los insectos por parte de las comunidades indígenas del oriente amazónico. 
Insectos usados como alimento por las comunidades indígenas. - En el oriente amazónico se reconocen diferentes especies de insectos empleadas como alimento. De acuerdo con su valor de uso las especies comestibles más importantes son el mojojoy Rhynchophorus palmarum (L.), la manivara Syntermes spinosus (Latreille), la hormiga arriera Atta laevigata (Smith), el tapurú Lusura chera (Drury), y a la avispa arrendajera Polybia liliacea (Fabricius) (Tabla 3).
Los entrevistados mencionaron de manera aislada, otros insectos comestibles como varios tipos de orugas de lepidópteros (gusanos), larvas de escarabajos y la diferenciación de hormigas nocturnas y diurnas. De acuerdo con el lenguaje o lengua de cada etnia indígena, los insectos comestibles reciben un nombre (Tablas 4 y 5). Las especies comestibles se pueden encontrar en el monte bravo, los rastrojos, chagras, potreros y en lugares con palmas caídas, o inclusive en los patios de las casas. Para su recolecta, simplemente se buscan manualmente en estos lugares o se puede emplear alguna técnica específica (Tabla 6).

Tabla 3. Especies de insectos comunes usadas como alimento por parte de las comunidades del oriente amazónico.

\begin{tabular}{|c|c|c|c|c|c|c|c|c|}
\hline \multirow{2}{*}{ Nombre científico } & \multirow{2}{*}{ Familia } & \multirow{2}{*}{ Orden } & \multirow{2}{*}{$\begin{array}{l}\text { Nombre } \\
\text { común }\end{array}$} & \multirow{2}{*}{ Nombre local } & \multirow{2}{*}{$\begin{array}{l}\text { Estado de } \\
\text { consumo }\end{array}$} & \multirow{2}{*}{ Modo de consumo } & \multicolumn{2}{|c|}{ Valor de Uso } \\
\hline & & & & & & & $U v_{1}$ & $U V_{2}$ \\
\hline $\begin{array}{l}\text { Rhynchophorus palmarum } \\
\text { (Linnaeus, 1758) }\end{array}$ & Curculionidae & Coleoptera & Mojojoy & $\begin{array}{l}\text { Neeĩnĩkowa } \\
\text { (Cubeo) }\end{array}$ & Larva & $\begin{array}{l}\text { Cocidos, tostados, } \\
\text { ahumados, crudos } \\
\text { (vivos) }\end{array}$ & 0.4 & 0.8 \\
\hline $\begin{array}{l}\text { Syntermes spinosus } \\
\text { (Latreille, 1804) }\end{array}$ & Termitidae & $\begin{array}{l}\text { Blattodea } \\
\text { (Isoptera) }\end{array}$ & Manivara & $\begin{array}{l}\text { Barijtjinawa } \\
\text { (Cubeo) }\end{array}$ & Adultos & $\begin{array}{l}\text { Cocidos, tostados, } \\
\text { crudos (vivos) }\end{array}$ & 0.7 & 0.8 \\
\hline $\begin{array}{l}\text { Atta laevigata } \\
\text { (Smith, 1958) }\end{array}$ & Formicidae & Hymenoptera & $\begin{array}{l}\text { Hormiga } \\
\text { arriera }\end{array}$ & $\begin{array}{l}\text { Meãwa Yupariwa } \\
\text { (Cubeo) }\end{array}$ & Adultos & $\begin{array}{l}\text { Tostadas, crudas } \\
\text { (vivas) }\end{array}$ & 0.7 & 0.3 \\
\hline $\begin{array}{l}\text { Lusura chera } \\
\text { (Drury, 1773) }\end{array}$ & Notodontidae & Lepidoptera & Tapurú & $\begin{array}{l}\text { Meñeñewua } \\
\text { (Carapana) }\end{array}$ & $\begin{array}{l}\text { Larvas } \\
\text { (orugas) }\end{array}$ & $\begin{array}{l}\text { Cocidos, tostados, } \\
\text { crudos (vivos) }\end{array}$ & 0.3 & 0.2 \\
\hline $\begin{array}{l}\text { Polybia liliacea } \\
\text { (Fabricius, 1804) }\end{array}$ & Vespidae & Hymenoptera & $\begin{array}{l}\text { Avispa } \\
\text { arrendajera }\end{array}$ & $\begin{array}{l}\text { Boborowa } \\
\text { (Cubeo) }\end{array}$ & $\begin{array}{l}\text { Larvas y } \\
\text { pupas }\end{array}$ & $\begin{array}{l}\text { Tostados, crudos } \\
\text { (vivas) }\end{array}$ & 0.06 & 0.1 \\
\hline
\end{tabular}

Tabla 4. Algunos nombres de insectos en lengua indígena de la etnia Carapaná.

\begin{tabular}{ll}
\hline Nombre en lengua & Significado \\
\hline Wõkoro & cucarrón comestible \\
Ňupuã & larva de escarabajo \\
Papiku & larva de escarabajo de guarumo \\
Yayaru & larva de escarabajo \\
Busamu & escarabajo de la chagra \\
Meneña & gusanito de guamo \\
Meñeñewua & tapurú de monte bravo \\
Bactiña & gusano comestible \\
Yepajã & larva de cucarrón \\
Buoza & larva de cucarrón pequeño de corteza de árbol \\
\hline
\end{tabular}

Tabla 6. Técnicas de colecta de insectos comestibles

\begin{tabular}{lll}
\hline $\begin{array}{l}\text { Tipo de insecto } \\
\text { comestible }\end{array}$ & Lugar de colecta & Técnica de colecta \\
\hline Tapurú & $\begin{array}{l}\text { Monte bravo, } \\
\text { rastrojo. }\end{array}$ & $\begin{array}{l}\text { Manualmente o haciendo } \\
\text { un encerrado. }\end{array}$ \\
Mojojoy & $\begin{array}{l}\text { Potrero, rastrojo, } \\
\text { miritisales. }\end{array}$ & $\begin{array}{l}\text { Cortar con hacha el tronco } \\
\text { de la palma y colectar } \\
\text { manualmente las larvas de } \\
\text { mojojoy. }\end{array}$ \\
Hormiga arriera & $\begin{array}{l}\text { Rastrojo, monte } \\
\text { bravo, chagra. }\end{array}$ & $\begin{array}{l}\text { Apertura de hueco pro- } \\
\text { fundo, uso de fuego para } \\
\text { acorralar a las hormigas. }\end{array}$ \\
Manivara & $\begin{array}{l}\text { Rastrojo, monte } \\
\text { bravo, chagras. }\end{array}$ & $\begin{array}{l}\text { Apertura de hueco cerca } \\
\text { del nido. Uso de fibra para } \\
\text { atrapar las termitas. }\end{array}$ \\
Avispa arrendajera & $\begin{array}{l}\text { Árboles en monte } \\
\text { bravo y chagras. }\end{array}$ & $\begin{array}{l}\text { Quema de nidos y poste- } \\
\text { rior recolecta manual en } \\
\text { la noche. }\end{array}$ \\
\hline
\end{tabular}

Tapurú. - El término tapurú es el nombre común asignado por las etnias indígenas del oriente amazónico, a diferentes tipos de orugas de lepidópteros o "gusanos" que se usan como alimento (Fig. 5). Generalmente se encuentran en árboles hospederos de diferentes especies. Existen diferentes especies de orugas comestibles en la región, a las que se les asigna un nombre específico en lengua indígena, de acuerdo con la planta hospedera en donde se encuentran (Tabla 5). Una de las más conocidas es la especie Lusura chera (Notodontidae: Nystaleinae), la cual es consumida durante su época de abundancia, entre agosto y septiembre (Fig. 5a). La forma más común para recolectar las orugas es manualmente, cuando son encontradas en las plantas o árboles hospederos. Únicamente se debe esperar a que las orugas bajen del árbol hospedero al suelo para recogerlas con la mano.

Para recolectar otras orugas comestibles, es necesario derribar el árbol hospedero. De acuerdo con los Cubeo, algunas orugas comestibles se les encuentra en el monte bravo y el rastrojo, por ejemplo, en el árbol de acaricuara Minquartia guianensis Aubl., donde se les hace un "encerrado" en la base del árbol utilizando hojas frescas en forma circular para que las orugas queden atrapadas en ese encierro. Los Yurití afirman que los gusanos comestibles como el tapurú solo se consigue en el monte bravo.

Mojojoy. - El término mojojoy es el nombre común de las larvas del escarabajo picudo Rhynchophorus palmarum (Curculionidae: Dryophthorinae), las 
cuales se alimentan de diferentes especies de palmas comunes de la región amazónica colombiana. La forma de recolectar las larvas es buscando dentro de las palmas derribadas de mirití o canangucho Mauritia flexuosa L. F., pataba Oenocarpus bataua Mart., y de ibacaba Oenocarpus bacaba Mart., con la ayuda de un hacha para romper la madera dura y extraer los especímenes de forma manual y luego recogerlos en una olla (Fig. 6). Después de dos o tres meses de tumbar la palma, se recoge o se "cosecha" el mojojoy. Al momento de tumbar la palma se hace un hueco para que el adulto hembra deposite los huevos dentro de la palma.
Hormiga arriera. - Las hormigas arrieras o culonas, pueden recolectarse en los rastrojos, en el monte bravo o en ocasiones se encuentran nidos en los patios de las casas. Una de las especies de hormiga comestible más comunes en la región es Atta laevigata (Formicidae: Myrmicinae). La técnica para recolectar hormigas consiste en ubicar un hormiguero (Fig. 7a) y con la ayuda de un palo al que se le saca punta, se hacen huecos profundos por donde saldrán las hormigas (Fig. $7 b$ ). Luego, en el hueco se introduce una varita larga de alguna rama, para que las hormigas comiencen a subir por ella (Fig. 7c, d). Poco a poco las hormigas van subiendo y cuando hay varias se procede a empujar las hormigas hacia un balde lleno de agua (Fig. 7e, f).

Tabla 5. Plantas hospederas de especies de orugas comestibles o "tapurú" en lengua Cubeo. Nombres comunes tomados de Cárdenas et al. (2007).

\begin{tabular}{|c|c|c|c|c|}
\hline Especie & & Nombre común & Nombre en Cubeo & Oruga en Cubeo \\
\hline \multirow{2}{*}{ Lauraceae } & Aniba sp. & Loiro negro & Joktñemikt & \multirow{6}{*}{ Jokłwa (tapurú) } \\
\hline & Aniba panurensis & Loiro & Jiadokt & \\
\hline \multirow{2}{*}{ Myristicaceae } & Virola calophylla & Vilora & Pojãborikt & \\
\hline & Vilora sp. & Vilora & KãKõdoakt & \\
\hline Vochyslaceae & Ruizterania sacculata & & Yaikt & \\
\hline Burseraceae & Dacryodes chimatensis & Ivapichuna & ktpekt & \\
\hline \multirow{2}{*}{ Burseraceae } & Dacryodes chimatensis & Ivapichuna & ktpekt & \multirow{2}{*}{ 甘turĩwã (oruga de capullo) } \\
\hline & Trattinnickia peruviana & Caraño & Chipekt & \\
\hline Olacaceae & Minquartia guianensis & Acararicura & Yapechimukt & Yapechimuwã \\
\hline \multirow{3}{*}{ Vochyslaceae } & Erisma laurifolium & Baboso & Tuakt & \multirow{4}{*}{ Okomiãdãwa } \\
\hline & Ruizterania sacculata & Baboso & Yaikt & \\
\hline & Erisma sp. & Arenillo & Cabokt & \\
\hline Apocynaceae & Aspidosperma excelsum & & Joetortmü & \\
\hline Moraceae & Ficus obtusifolia & & Vedamu & Vedawa \\
\hline Lauraceae & Aniba panurensis & Loiro & Jiadokt & \multirow{5}{*}{ Doviwa } \\
\hline Vochyslaceae & Ruizterania sacculata & & Yaikt & \\
\hline \multirow{3}{*}{ Sapotaceae } & \multirow{3}{*}{ Pouteria bachniana } & \multirow{3}{*}{ Lulo de monte } & Yukukt & \\
\hline & & & Macabetacakt & \\
\hline & & & Meturiöwakt & \\
\hline Monimiaceae & Siparuna micrantha & Palo corteza & Takt & Takajedowa \\
\hline \multirow{2}{*}{ Cecropiaceae } & Pourouma minore & & Bobotomobü & \multirow{2}{*}{ Ñemikajedowa o biorowa } \\
\hline & Pourouma cucura & & Pujikamu & \\
\hline Sapotaceae & Poutria ucuqui & Ucuqui & Pacorokt & \multirow{2}{*}{ Uboabt } \\
\hline Icacinaceae & Poraqueiba sericea & Umarí & Mamtkt & \\
\hline Vochyslaceae & Erisma laurifolium & Baboso & Tuakt & \multirow{2}{*}{ Jłmeküwa } \\
\hline Sapotaceae & Pouteria bachniana & & Yucukt & \\
\hline Euphobiaceae & Hevea guianensis & Siringa & Yekakt & \multirow{3}{*}{ Kedavichiwa } \\
\hline \multirow{2}{*}{ Sapotaceae } & Pouteria bachniana & & Yucukt & \\
\hline & Ecclinusa cf. guianensis & & Pikakt & \\
\hline \multirow{2}{*}{ Mimosaceae } & Inga edulis & Guamo bejuco & Menekt & \multirow{2}{*}{ Kuãbejorłwa } \\
\hline & Cedrelinga cateniformis & Yacayaca & Mujẽkt & \\
\hline \multirow{4}{*}{ Mimosaceaea } & Inga acuminata & Guamo de yaripa & Pinpinmenekt & \multirow{4}{*}{ Jĩmiãwa } \\
\hline & Inga spectabilis & Guamo machete & Abacamenekt & \\
\hline & Inga sp. & Guamo perro de agua & Jiadavimenekt & \\
\hline & Inga sp. & Guamo de guio & Aĩmenekt & \\
\hline Fabaceae & Monopteris uaucu & Avina & Avinakt & Makupawa \\
\hline \multirow{2}{*}{ Myristicaceae } & Iranthera triconis & Molinillo & Utujicamu & \multirow{2}{*}{ Mujãmevã } \\
\hline & Virola calophylla & Vilora & Pojãborikt & \\
\hline Moraceae & Helicostylis tomentosa & & Potatakt & \multirow{2}{*}{ Pöjãwã } \\
\hline Mimosaceae & Inga edulis & Guamo bejuco & Menekt & \\
\hline
\end{tabular}



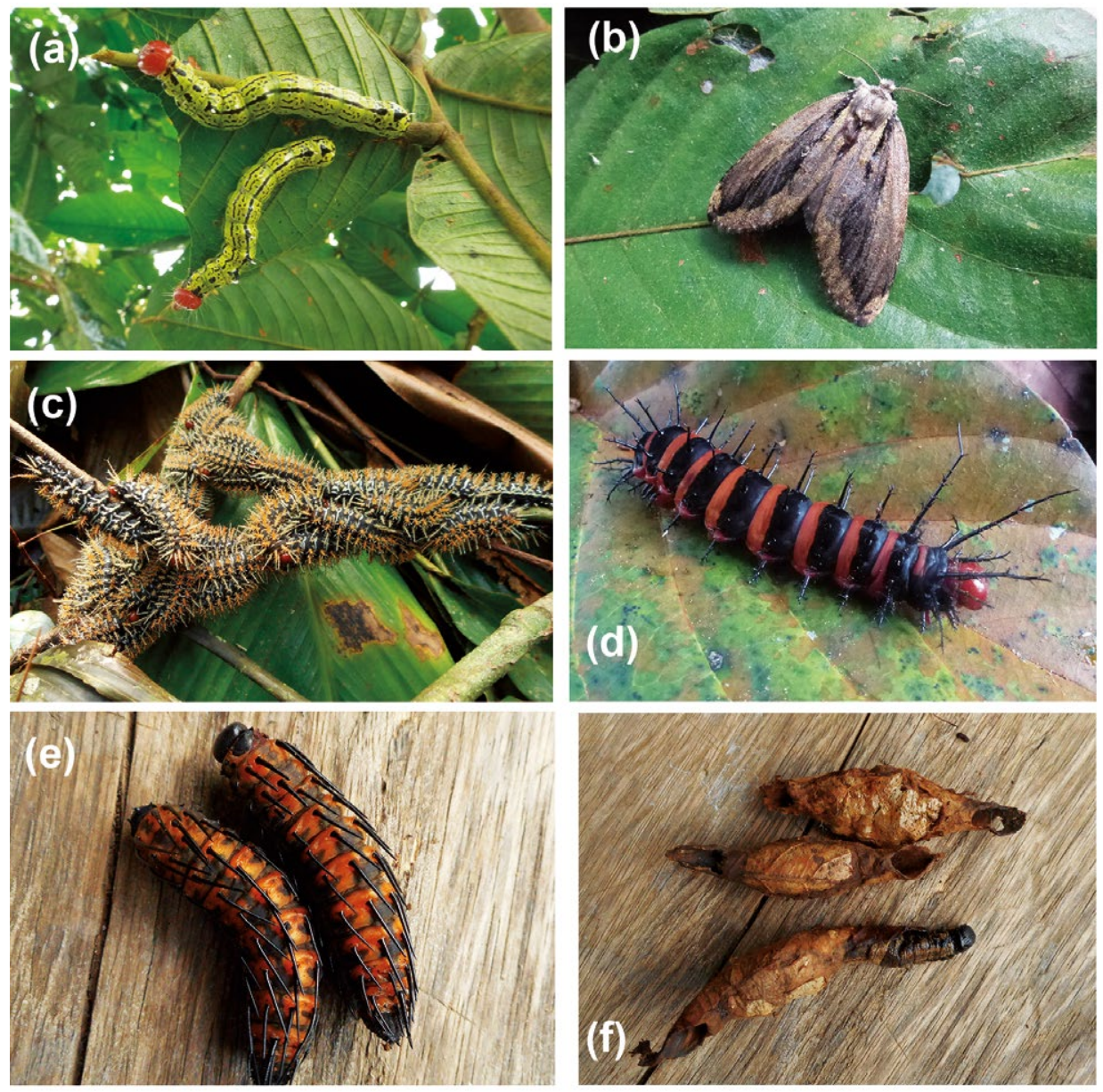

Figura 5. Orugas comestibles en el oriente amazónico. a.- Oruguita de guamo (Jĩmiãwa) - Lusura chera (Drury), Notodontidae, $b$. Adulto de Lusura chera (Drury) c. Tapurú (Jokłwa) - Saturniidae: Hemileucinae, d. Tapurú (Yapechimuwã) - Saturniidae: Hemileucinae, e. Tapurú (Kuãbejorłwa) - Saturniidae: Ceratocampinae, $\mathrm{f}$.

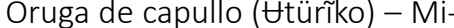
mallonidae.

(a)
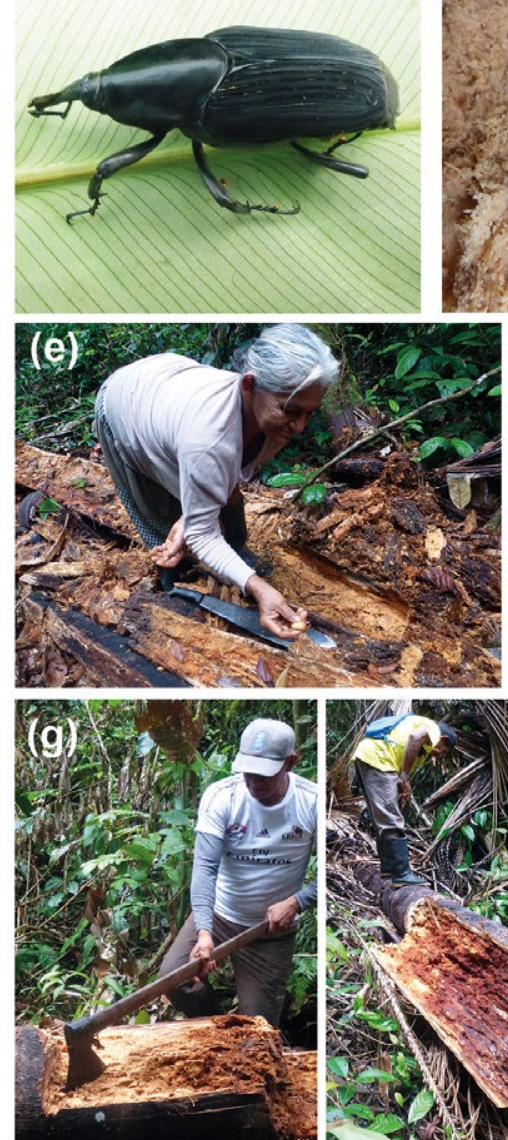
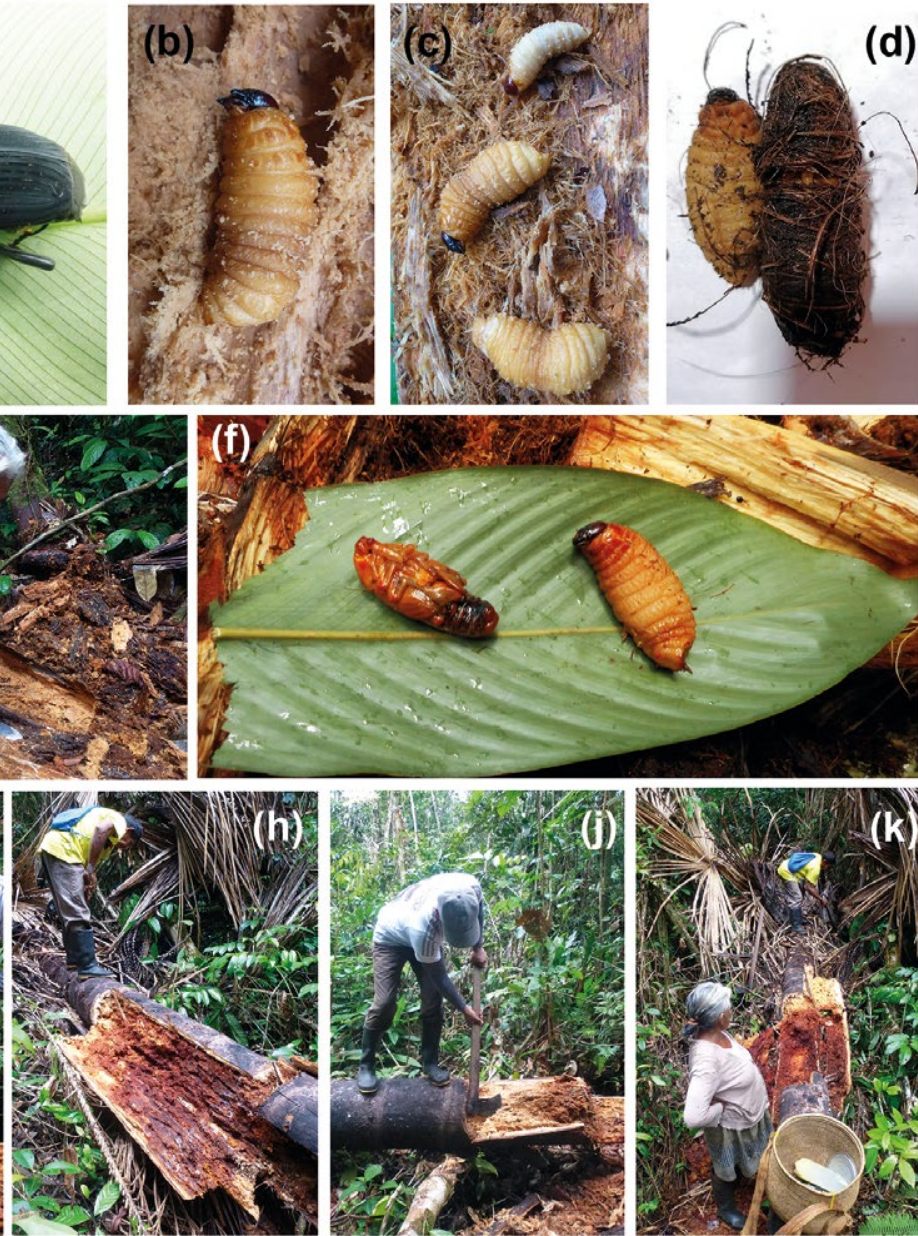

(d)

Figura 6. Recolecta de mojojoy. a. Adulto de Rhynchophorus palmarum (Coleoptera: Curculionidae), b, c. Larvas de R. palmarum, d. Larva y capullo de mojojoy, e. Colecta manual de mojojoy, f. Pupa y larva de mojoy de mirití, $g-k$. Técnica de recolecta de mojojoy. 

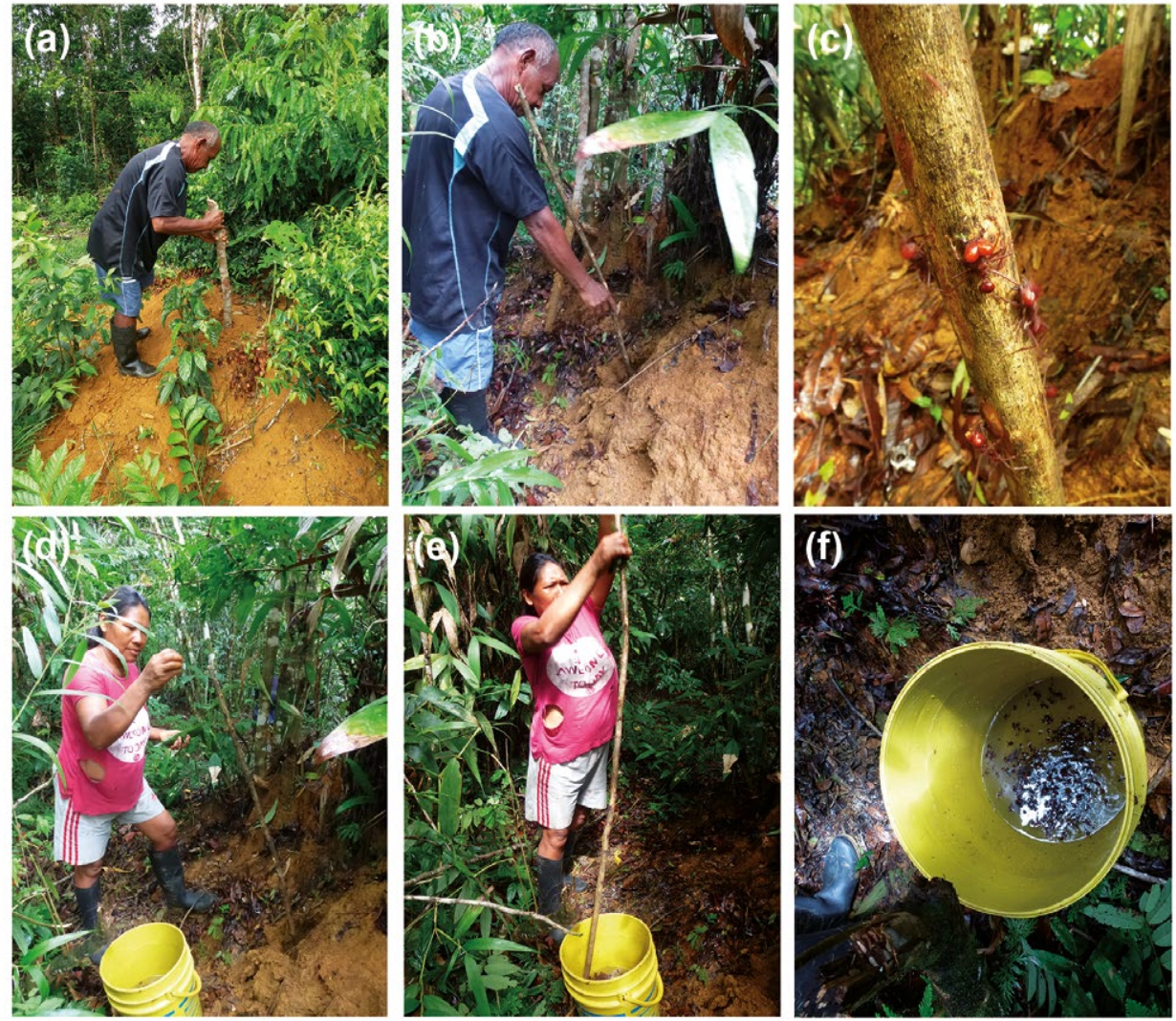

Figura 7. Colecta de hormiga arriera. a, b. Localización del nido. c. Hormigas subiendo en el palo insertado en el nido. d, e, f. Colecta manual de hormigas y transporte.

De acuerdo con los Cubeo, otra técnica para recolectar hormigas en la noche es "hacer un hoyo de 30 $\mathrm{cm}$ de profundidad y de aproximadamente $25 \mathrm{~cm}$ de diámetro en la superficie de la tierra con la ayuda de un machete a unos 6 metros de distancia del nido. Luego se prende una llama con la planta del turi (Eschweilera rufifolia S.A. Mori, Lecythidaceae) para que las hormigas lleguen hacia la luz de la candela y caigan en el hueco, luego las hormigas se van recogiendo en una olla". El turi es una planta útil de la región del Vaupés, usada también para la fabricación de canastos (Osorno et al. 2014).

Según los Cubeo, otra técnica empleada para recoger hormigas es construir un "llerado", una sencilla estructura a manera de andamiaje: -"hecho con palos gruesos que resista el peso de la persona, así se recoge a las hormigas culonas, estando sobre el llerado y los nidos están debajo de ella". Otra forma de recolecta consiste simplemente en esperar cerca del nido para recoger las hormigas con la mano cuando salen a volar. De acuerdo con los Tatuyo, es recomendable que cuando se busquen hormigas en los nidos, la persona se coloque botas pantaneras para protegerse de las mordeduras de los soldados, que pueden causar profundas heridas.

Manivara. - La palabra manivara se refiere a las termitas o comejenes comestibles de la especie Syntermes spinosus (Termitidae: Syntermitinae). Generalmente se recolectan en el monte bravo, rastrojo y chagras (Fig. 8). Los Cubeo y Macuna emplean una técnica específica para colectar manivara para el consumo, que consiste en abrir un hueco cerca del nido principal y esperar que salgan en cantidad, luego se toma una fibra blanda de una planta del monte para que las termitas las muerdan. Luego de esperar un poco, se recoge la fibra en donde las termitas están sujetadas y se va recogiendo en una olla con agua. Los Tatuyo coinciden en emplear esta misma técnica, previamente identificando el camino de las termitas cerca del nido principal.

Avispa arrendajera. - También llamada avispa boburona corresponde a la especie Polybia liliacea (Vespidae: Polistinae), cuyas larvas y pupas son consumidas por etnias como los Cubeo y Guanano (Fig. 9). Pueden recolectarse en el monte bravo y en las chagras buscando en los árboles donde se hallan estos insectos. Para poder recolectarlos es necesario quemar muchas veces el nido y luego ir en la noche y recolectar las larvas manualmente.

Las avispas de nidos grandes reciben un nombre en lengua Cubeo como Bejowa, Boborowa y Büyawa (Polybia spp.). Las Büyawa construyen sus avisperos en las copas de los árboles de bosques primarios y secundarios. Para consumir las larvas de estas avispas, los colectores derriban el árbol hospedero para poder recoger el avispero. El tiempo de recolecta debe ser muy rápido, apenas cuando el árbol cae al suelo. Es necesario cortar rápidamente el avispero y alejarse con él evitando las picaduras de las avispas. 

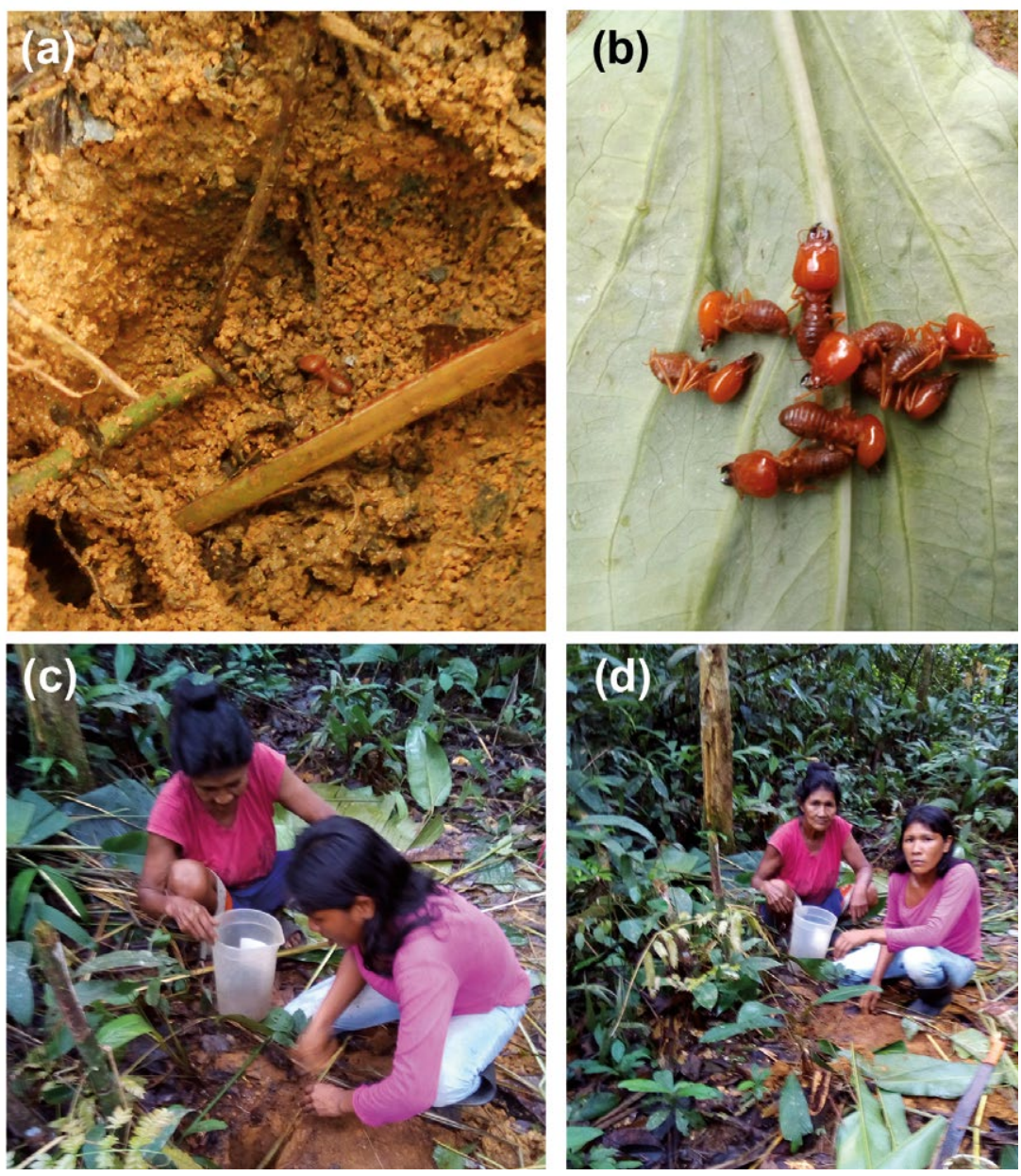

Figura 9. Avispas comestibles. a, b. Adulto de la avispa arrendajera Polybia liliacea, c. Avispero Boborowa (Cubeo), d. Avispero Abuju viakawa (Cubeo), e. Avispero Ñenenewa (Cubeo), f. Técnica de colecta de avispas comestibles. (Fotos: William González).
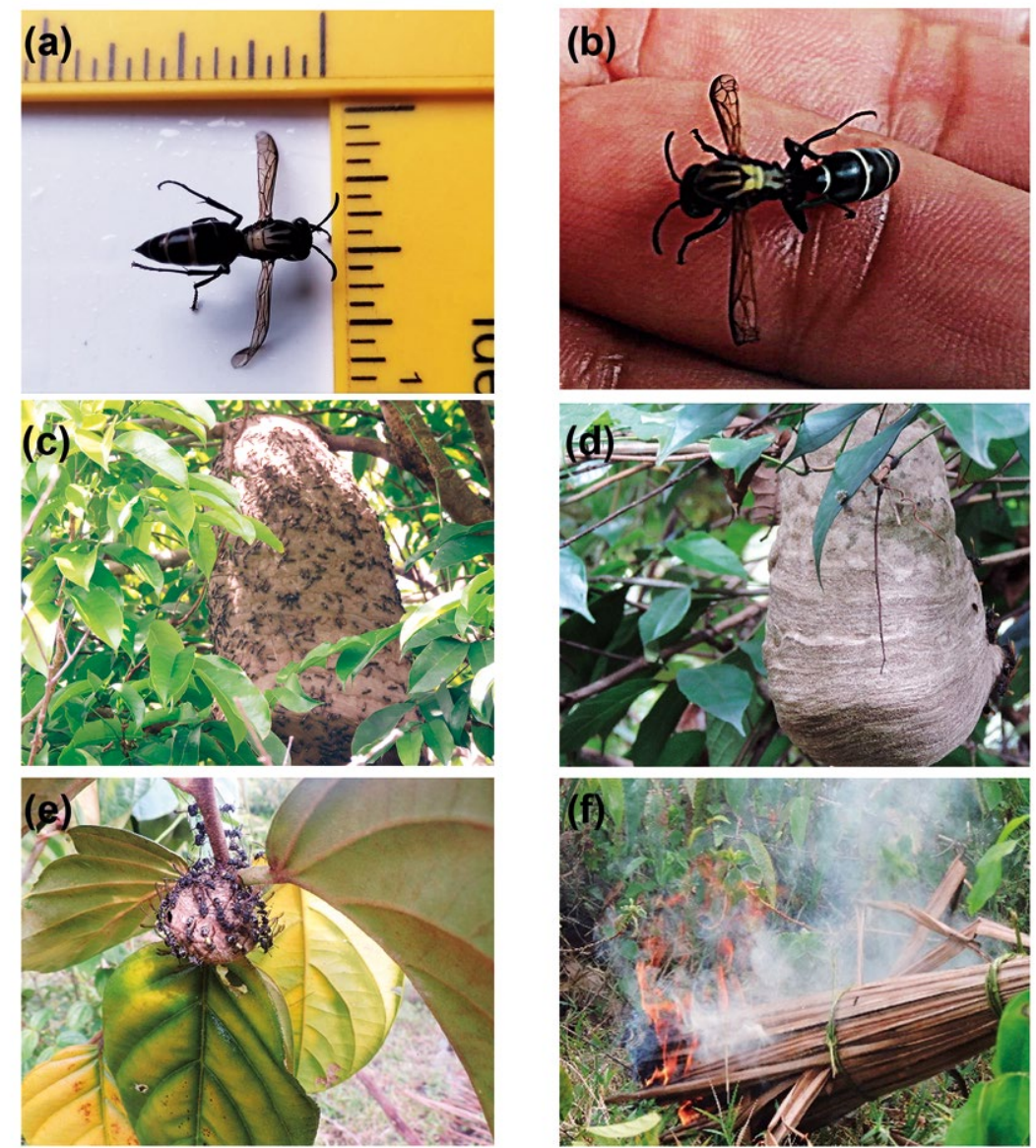

Figura 8. Comején comestible. a. Nido de manivara. b. Manivara - Syntermes spinosus. (Blattodea: Termitidae), c y d. Colectoras en busca de manivara. 
Para los avisperos Bejowa y Boborowa, ubicados en ocasiones hasta 5 metros de altura en los árboles, generalmente las avispas son ahuyentadas con humo. Esta técnica consiste en seleccionar una vara de tres a cinco metros y amarrar en la punta las hojas secas de las palmas seleccionadas y encender fuego (Fig. 9f). Cuando el humo empieza a producirse, es necesario acercar la vara rápidamente hacia el avispero. Existen otras avispas como las Ñenenewa (Polybia sp.), a las cuales se le puede ahuyentar utilizando una camisa sudada amarrada también con una vara, y acercándola al avispero.

Calendario ecológico. - La abundancia de insectos comestibles se presenta en una época especifica del año, y de acuerdo con las diferentes etnias entrevistadas, es recomendable recolectar los insectos a determinadas horas del día con el fin de que la jornada, la cual dependiendo del insecto comestible requiere de cierto tiempo, sea exitosa. En general, una buena hora para recolectar insectos comestibles es a partir de las 9:00 am (Tabla 7).

Tapurú. - El tapurú se puede recolectar en diciembre a inicios del verano. De acuerdo con los Macuna, Cubeo y Carapana, son más frecuentes entre agosto y septiembre en época de invierno o verano corto, y esporádicamente en junio. Los Tatuyo afirman que la época de gusanos comestibles es entre junio y septiembre. Para los Yurutí, el tapurú es abundante entre agosto y septiembre hasta inicios de octubre generalmente en todos los años, aunque no siempre aparecen. Según los Carapana, la hora apropiada para recolectar tapurú es a partir de las 7 de la mañana. Los Cubeo sugieren recolectar tapurú desde las 9:30 de la mañana y a las 12:30 pm, en un día preferiblemente soleado. El tiempo de recolecta del tapurú es de 30 minutos en los árboles y plantas herbáceas hospederas. En ocasiones de una a dos horas dependiendo de la distancia a la que se encuentra el lugar de recolecta. En la época de orugas, puede programarse una recolecta de seis horas semanales, dos veces por semana.

Mojojoy. - De acuerdo con los Macuna, Cubeo, Desano y Waniba, el mojojoy se recolecta en cualquier época del año para el consumo, y a cualquier hora del día, preferiblemente entre las 9:00 y 10:30 de la mañana en días soleados. Según los Siriana, el mojojoy se recolecta en diciembre para las festividades que se realizan en ese mes. Los Waniba señalan que, la recolección del mojojoy depende de "cuando se nota que la palma muerta tiene indicios de tener mojojoy", lo que estaría relacionado con el grado de descomposición del tronco de la palma. Los Desano indican que cuando se tumban palmas de milpesos o de mirití, se pueden obtener larvas de mojojoy después de 3 o 4 meses para el consumo. Para los Macuna y Cubeo, si el lugar es cercano, el tiempo de recolecta es de 30 minutos. Por lo general, la jornada puede durar de 2 a 3 horas en busca de mojojoy, en ocasiones hasta 4 horas. Según los Macuna es posible recolectar en una jornada, de 1 a 2 kg de larvas de mojojoy.

Hormiga arriera. - De acuerdo con los Cubeo, las hormigas arrieras se recolectan en abril preferiblemente en un rango que puede ir desde las 11:00 am a las 4:00 pm, en temporada de lluvia o al inicio del invierno. Las hormigas nocturnas en marzo, abril y los primeros días de mayo a las 4:00 o 5:00 am. Las hormigas diurnas se pueden recolectar a finales de abril o durante la semana santa cuando vuelan las hormigas. Según los Tatuya, a las hormigas se les debe recolectar desde las 11:30 am, en la época del inicio de la lluvia del año. Los Guanano aseguran que las hormigas arrieras pueden obtenerse anualmente solamente al inicio del invierno en los meses de abril durante el primer vuelo, y en junio durante el segundo vuelo, que se puede observar durante las 11:00 am a las 4:00 pm. La recolección de las hormigas culonas generalmente puede durar de 3 a 4 horas, en ocasiones hasta 5 horas. Algunos Cubeo sugieren que el tiempo que se le dedica a recoger hormigas es desde las 10 am hasta las 4 pm (Tabla 7). Algunas jornadas pueden ser más cortas, desde la 1 pm hasta las 3 pm, preferiblemente en días soleados.

Manivara. - La manivara se puede recolectar en cualquier época del año, generalmente cuando hay luna llena o luna creciente. Pueden ser frecuentes en abril y septiembre. Según los Cubeo, una buena hora del día para recolectar manivara es en la mañana, después de las 8 am o desde la 9:30 a 10:30 am. De acuerdo con los Macuna, es recomendable salir a recolectar manivara temprano, antes de la 8 am y por la tarde a la $3 \mathrm{pm}$. También si el nido de la manivara se encuentra cerca de la casa o en la chagra, se recoge en la noche. El tiempo empleado para recolectar manivara es de 1 hora. Los Cubeo emplean de 2 a 3 horas, tiempo de colecta suficiente para llevar el alimento a la familia (Tabla 7). Los Macuna recogen

Tabla 7. Calendario ecológico de insectos comestibles más importantes en el oriente amazónico.

\begin{tabular}{llll}
\hline Tipo de insecto comestible & Época de abundancia & Hora apropiada de recolecta & $\begin{array}{l}\text { Tiempo promedio empleado } \\
\text { en la recolecta }\end{array}$ \\
\hline Tapurú & Agosto, septiembre y octubre & 7 am a 12:30 pm & 30 min a 2 horas \\
Mojojoy & Durante todo el año & $9: 00$ a 10:30 am & 2 a 3 horas \\
Hormiga arriera & Marzo - Abril & $11: 00 \mathrm{am}$ a 4:00 pm & 3 a 4 horas \\
Manivara & Durante todo el año & $9: 30$ a 10:30 am & 2 a 3 horas \\
Avispa arrendajera & Durante todo el año & Cualquier hora del día & 1 a 2 horas \\
\hline
\end{tabular}


manivara entre 40 minutos hasta 2 horas, tiempo adecuado para conseguir suficientes para el consumo, mientras que los Guanano pueden emplear entre 1 a 2 horas.

Avispa arrejandera. - Generalmente, las avispas comestibles pueden estar disponibles durante todo el año, y dependiendo de la distancia en donde se ubique el avispero, la jornada de recolecta puede durar entre 1 y 2 horas (Tabla 7). De acuerdo con los $\mathrm{Cu}-$ beo, la mejor época para recolectar avispas es durante la luna llena.

Uso y preparación de insectos comestibles para consumo. - Existen diferentes formas tradicionales de preparar el alimento de acuerdo con el tipo de insecto comestible. Según los Caparana, los insectos son un alimento que acompaña muy bien cualquier tipo de comida. Los Cubeo recomiendan que: "A los insectos se les debe preparar muy bien para que sean muy nutritivos".

La mayoría de los entrevistados de las diferentes etnias consultadas coinciden en afirmar que el consumo de insectos no forma parte de la alimentación diaria. Generalmente está limitado a la época en la que se "consiguen" o "aparecen", es decir, solamente se incluyen insectos en la alimentación cuando sus poblaciones abundan, lo que está directamente relacionado con su calendario ecológico. Algunas etnias como los Cubeo y Tatuyo consideran cotidiano el consumo de manivara y mojojoy en su alimentación. Algunos Cubeo afirman que si se quiere tener quiñapira de manivara es necesario ir a recogerlas cada ocho días, mientras que los Caparana mencionan que los insectos para consumo siempre se consiguen en las chagras como el caso de la manivara.

Tapurú. - De acuerdo con los Cubeo, para la preparación de los gusanos comestibles, es necesario lavarlos muy bien. Luego se hierve agua con mucha leña. Cuando el agua ha hervido se agregan los tapurús vivos y se cocinan bien para que se les quite el "veneno", durante 45 minutos a 1 hora o 1 hora y 30 minutos. Luego se separan de la olla y se enfrían para comer frescos o tostados, y se pueden poner a ahumar encima del fogón. También se pueden tostar en el tiesto y posteriormente se pasan por un molino para consumir. El tapurú se puede acompañar con fariña, casabe, ají fresco, yuquitaña (ají molido), con jugo de piña o plátano. Los Guanano sugieren cocinar el tapurú durante 1 hora y 45 minutos para luego consumir acompañado de casabe, fariña, arroz y un buen jugo de piña o asai. Los Yurutí mencionan que los gusanos comestibles primero deben ser hervidos solamente en agua y luego se vierten en otra olla con agua hirviendo, para después separarlos del fogón. El agua usada debe eliminarse para después lavar muy bien los gusanos con agua limpia, y así se pueden empezar a cocinar durante un tiempo de cocción de 1 hora para comer acompañado de casabe y fariña.

Mojojoy. - Para los Macuna, Siriana y Cubeo, el mojojoy se puede consumir fresco, es decir vivo y recién extraído del tronco de la palma, aunque la mejor manera es cocinarlo. Primero las larvas deben lavarse muy bien. Luego en una olla se cocinan con agua durante 20 a 45 minutos. Los Macuna sugieren un menor tiempo de cocción que va de 7 a 15 minutos. Las larvas del mojojoy también se pueden preparar ahumadas, fritas, asadas en una paila o tostadas en el tiesto para después molerlas y consumirlas. Cuando se prepara ahumado, el mojojoy se envuelve en hojas de palma, y dura en ese proceso aproximadamente 30 minutos en el fogón. Generalmente, el mojojoy está listo para su consumo cuando las larvas están doradas, y se puede acompañar con casabe, fariña, ají molido, jugo de piña o jugo de la temporada como el wasay o asaí (Euterpe oleracea Mart., Arecaceae), o milpesillos. Los Desano preparan el mojojoy ahumado (moquiado) o tostado durante 35 minutos y lo consumen acompañado de casabe, fariña y en ocasiones con arroz.

Hormiga arriera. - Al igual que el mojojoy, la hormiga arriera se puede comer fresca o viva. El proceso de preparación de la hormiga es bastante simple. Se pueden tostar o fritar. Cuando se tuestan se utiliza el tiesto. Pueden durar hasta 45 minutos en el proceso, pero si se desea consumir molida, es necesario tostar durante 50 minutos a 1 hora. Para poder consumirlas "blanditas", los Cubeo sugieren tostar las hormigas en el tiesto de 15 a 20 minutos y acompañarlas con casabe y fariña.

Manivara. - Como el mojojoy y la hormiga arrierra, la manivara también puede consumirse viva. Para cocinarlas deben lavarse bien con agua limpia y tratando de extraer el barro que tienen dentro del cuerpo. Existen dos formas de preparar la manivara. La primera es cocinarlas en el caldo de quiñapira, un plato típico de la región a base de pescado y ají. Dentro del caldo, la manivara se puede cocinar durante una hora y media para que suelte el "sabor", para luego consumir acompañado de casabe y fariña. Otra forma de preparar la manivara es tostarla en el tiesto con suficiente leña. Se ponen a secar en el tiesto caliente durante una hora revolviéndola constantemente para evitar que se quemen. Cuando están bien secas o tostadas, se pasan al molino o pilón para comer con casabe y fariña, o también mezclado con un caldo de pescado (quiñapira). Los Tatuyo emplean 45 minutos para cocinar la manivara y la acompañan con pescado y ají fresco. Los Desano sugieren que la manivara siempre tiene que acompañar al plato de pescado con suficiente ají o consumir el caldo de pescado con manivara para comer acompañado de casabe y fariña.

Avispa arrendajera. - Las larvas de las avispas se pueden consumir vivas o frescas, o también tostadas. Para su preparación, se recolecta el avispero y se separan los discos donde están las larvas de las avispas (Fig. 10). Luego se asan en candela con poca llama los discos seleccionados, se coloca una hoja grande, que puede ser de plátano o platanillo para recoger las larvas de las avispas. También se pueden trans- 
portar en un balay, cesto tejido con fibras de guarumo (Cecropia peltata L.) o chambira (Astrocaryum chambira Burret). Las pupas y larvas de avispas se pueden acompañar con casabe.

Comercialización de insectos comestibles en el oriente amazónico. - Los insectos que se comercializan en Mitú son la manivara, el mojojoy, el tapurú y la hormiga arriera. Se venden en la plaza de mercado del Puerto de San Victorino en Mitú (Fig. 11). Cada producto proviene de un determinado lugar y tiene un precio, calidad y condiciones de venta específicas para su comercialización. Los comerciantes, que pertenecen a etnias como Cubeo, Tatuya y Siriana, ofrecen un tipo de insecto comestible, mientas que otros pueden ofrecer varios productos. Generalmente los compradores son los mismos indígenas que provienen de diferentes comunidades, aunque algunos mestizos también se interesan por los insectos comestibles. Algunos factores deben tenerse en cuenta para que el producto pueda ser vendido.
Tapurú. - El tapurú que se vende en la plaza de mercado proviene de las mismas comunidades en donde viven los comerciantes, generalmente recolectados en monte bravo y en las plantas o árboles hospederos. Se vende durante agosto, septiembre y algunos días de octubre, época del año en donde son abundantes. Los compradores son los parientes, vecinos o inclusive los pescadores de varias comunidades indígenas que usan las orugas para su oficio. El tapurú se ofrece en "nidos" que contienen varias orugas. Los nidos se reconocen como un estuche de seda que envuelve las orugas del tapurú (Fig. 12). El precio de venta depende del tamaño del nido. Los nidos más pequeños cuestan $\$ 5000$ (\$1.5 USD) y tienen un tamaño aproximado de $20 \mathrm{~cm}$. Los más grandes entre $\$ 10000$ y $\$ 15000$ pesos (\$2.5 - \$4 USD) y tienen un tamaño de 30 a $50 \mathrm{~cm}$. Las orugas o "gusanos" se ofrecen siempre frescos, que se pueda observar que las larvas de tapurú están vivas.
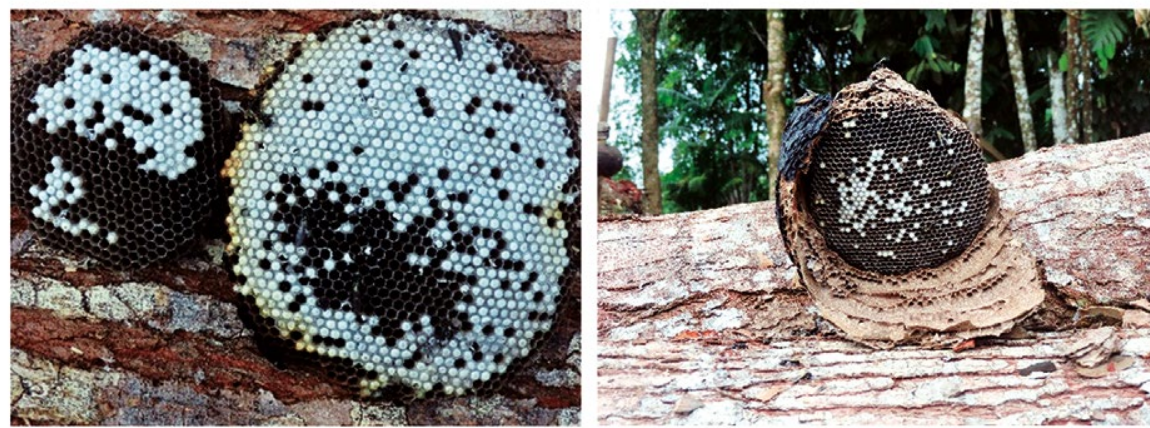

Figura 10. Discos de avisperos empleados para la extracción y consumo de larvas y pupas de avispas comestibles. Fotos: William González.
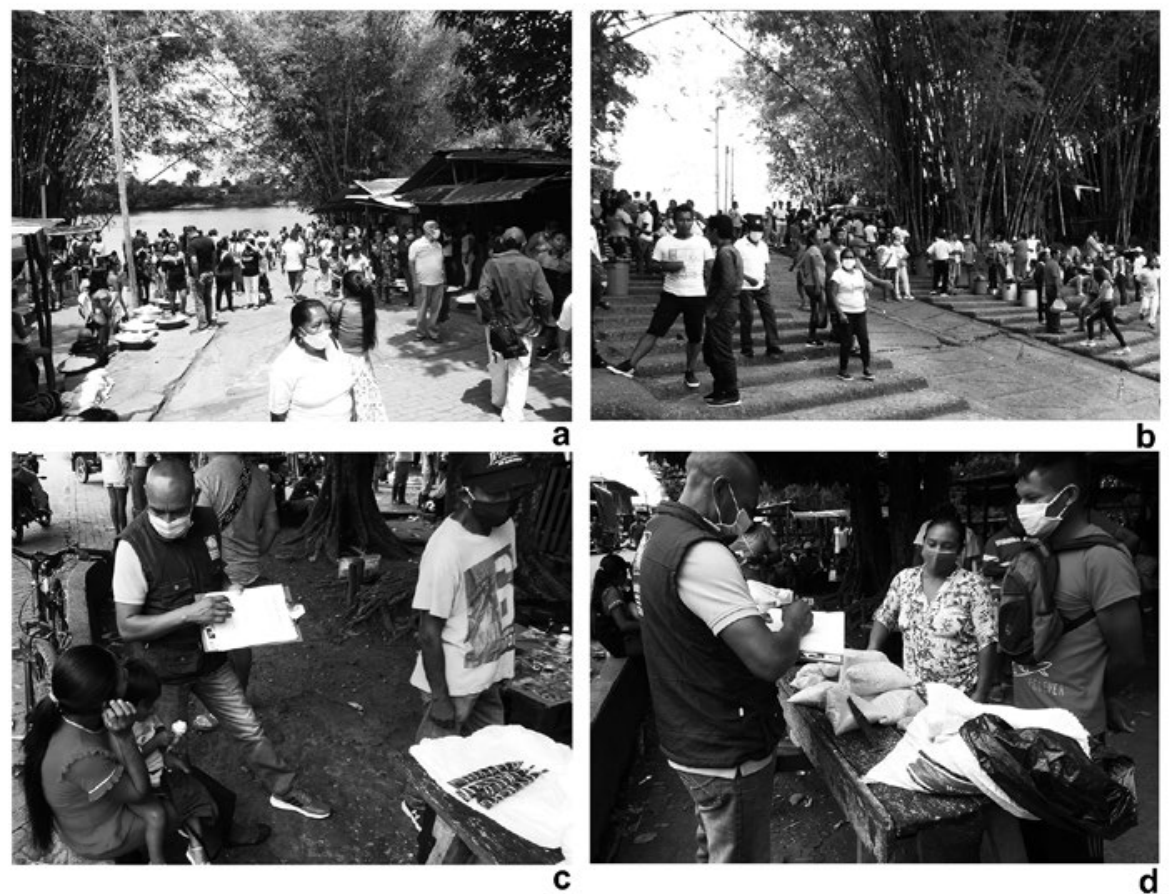

Figura 11. Mercado del puerto San Victorino, Mitú, Vaupés. a, b. Día de actividad comercial en el mercado. c, d. Entrevistas a comerciantes que ofrecen insectos comestibles. 

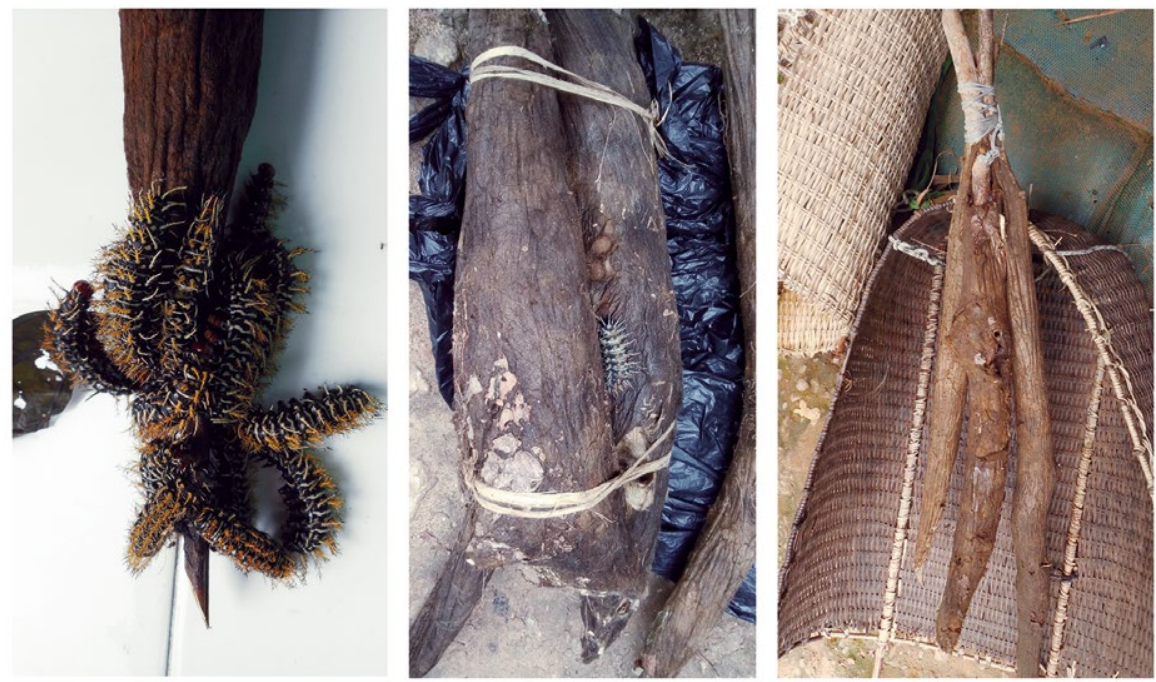

Figura 12. Formas de comercialización de orugas comestibles o tapurú.

Mojojoy. - El mojojoy se ofrece en la plaza de mercado del puerto de San Victorino del Mitú generalmente dos veces al año, o cuando exista disponibilidad. El producto se obtiene de las palmas caídas y secas de mirití, ibacaba o de milpesillos en el monte bravo. Los compradores son los indígenas y algunos mestizos ("blancos"). Las larvas de mojojoy se venden en envueltos. Para la venta, el mojojoy se recolecta un día antes de ofrecer el producto en la plaza de mercado. Los envueltos se hacen en el día anterior con hojas de palma y bien amarrados, y se ofrecen bien ahumados y no tan secos, con buena grasa para que tengan un aspecto aceitoso, lo que les gusta a los compradores. De acuerdo con los vendedores de la etnia Cubeo, "el mojojoy siempre se vende en 'envuelto' con hojas de milpesillos para conservar su grasa" (Fig. 13). A los compradores les gusta bien ahumado, no debe estar muy tostado ni seco, pero con buena cantidad de grasa acumulada dentro del envuelto, por lo cual los envueltos deben ser bien amarrados con cuerdas de lianas. Otros compradores prefieren el mojojoy recién colectado, vivo y fresco, lo que asegura que se pueda ver la calidad del producto. Estos también se venden en envueltos de hojas de palma de milpesillos.

Cada envuelto debe traer buena cantidad de larvas de mojojoy ya que el comprador revisa el contenido antes de llevarse el producto. Cada envuelto puede tener de 8 a 24 larvas y su precio varía entre $\$ 4000$ a $\$ 5000$ pesos ( $\$ 1$ - \$1.5 USD). Puede venderse también a $\$ 3.000$ pesos ( $\$ 50$ USD) con 16 unidades. Otra forma de vender el mojojoy es empacando las larvas vivas o ahumadas, en bolsas plásticas trasparentes de aproximadamente 200 gramos. El precio de cada bolsa es de $\$ 5000$ pesos ( $\$ 1.5$ USD).

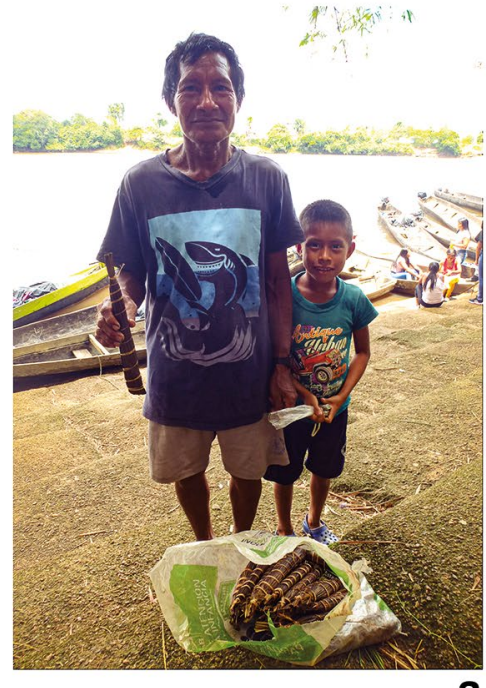

a

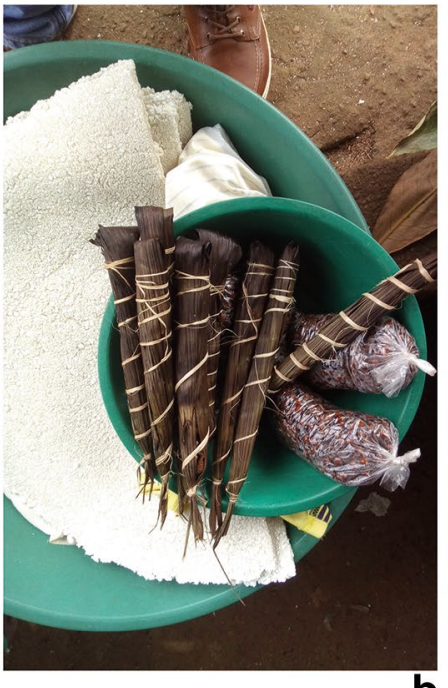

b

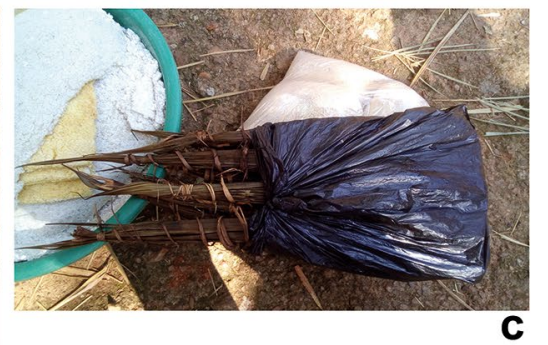

C

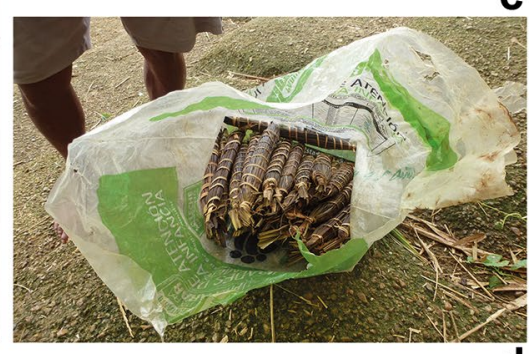

d

Figura 13. Comercio de mojojoy en el puerto de San Victorino. a. Comerciante ofreciendo mojojoy. b, c, d. Envueltos de mojojoy para la venta. 
Hormiga arriera. - Las hormigas provienen de los nidos encontrados en el monte bravo, las chagras y los rastrojos. Los compradores son los mismos habitantes de las comunidades que visitan la plaza de mercado de San Victorino de Mitú para obtener el producto. Las hormigas se ofrecen empacadas en frascos de plástico. Pueden ofrecerse frescas o vivas o tostadas y bien crocantes. El precio de venta por frasco es de $\$ 10000$ a $\$ 15000$ pesos ( $\$ 2.5$ - \$4 USD).

Manivara. - La manivara que se comercializa en la plaza de mercado de San Victorino de Mitú, es recolectada de los termiteros que se encuentran en el monte bravo, el rastrojo y las chagras cercanas a las casas de las comunidades. Generalmente se vende con frecuencia durante todo el año, dos veces cada mes, una o dos veces a la semana durante el año, o cada vez que los comerciantes requieren visitar la plaza de mercado para comprar otros productos y llevar a sus comunidades. Para la venta, la manivara debe ofrecerse bien fresca ya que, si se pasa de tiempo, puede producir un olor muy fuerte y así el producto no se vende. La manivara debe recolectar- se en la tarde del día anterior, o en la mañana del mismo día, para que sea fresca. No es recomendable exponerlas por mucho tiempo al sol, ya que pueden dañarse fácilmente. Se deben lavar bien y se dejan en una olla con agua tibia. Luego se secan y se empacan en bolsas plásticas para ofrecer en el mercado (Fig. $14 \mathrm{a}, \mathrm{b}, \mathrm{c})$. También pueden venderse bien tostadas, lo que facilita que el comprador las pueda moler para el consumo. Algunos comerciantes, después de lavar las termitas, agregan un poco de sal y luego se envuelven en bolsas plásticas. De acuerdo con los $\mathrm{Cu}$ beo, la manivara fresca recién sacrificada debe estar bien lavada, ya que muchos compradores las prefieren ver frescas. Otros compradores las prefieren medio tostadas.

Cada bolsa se puede llenar con una taza de manivara, lo que puede corresponder a aproximadamente 120 a 160 gramos de termitas frescas (Fig. 14d, e, f). La bolsa se vende por un precio de $\$ 2000$ o $\$ 3000$ pesos ( $\$ 50$ - \$1 USD), y generalmente se comercializan entre 10 y 25 bolsas de manivara por jornada de venta.
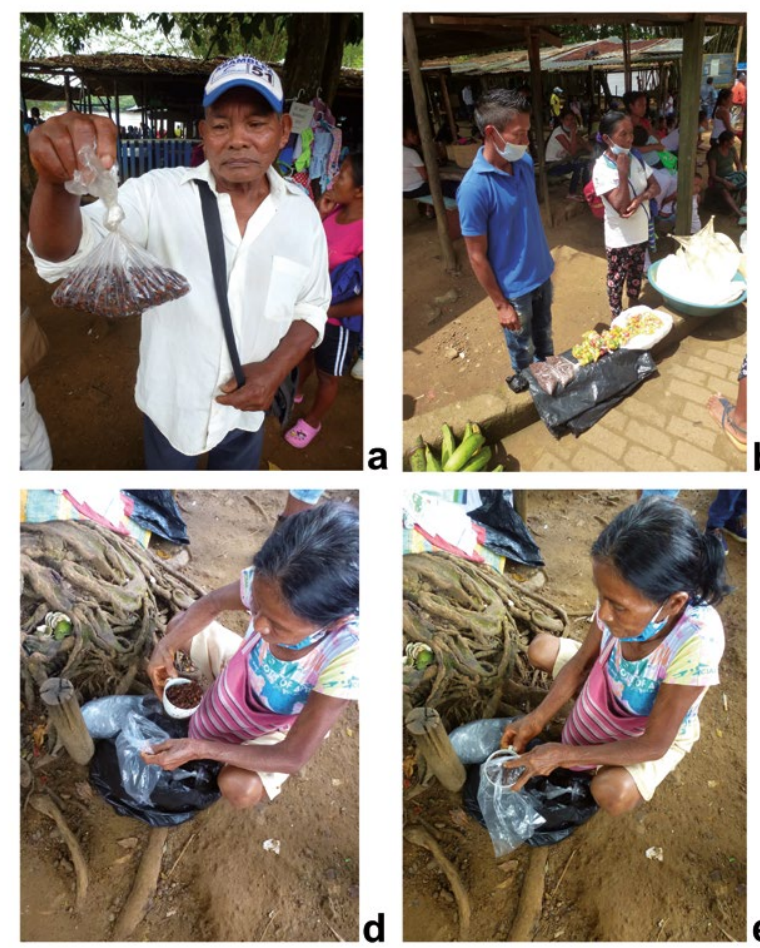
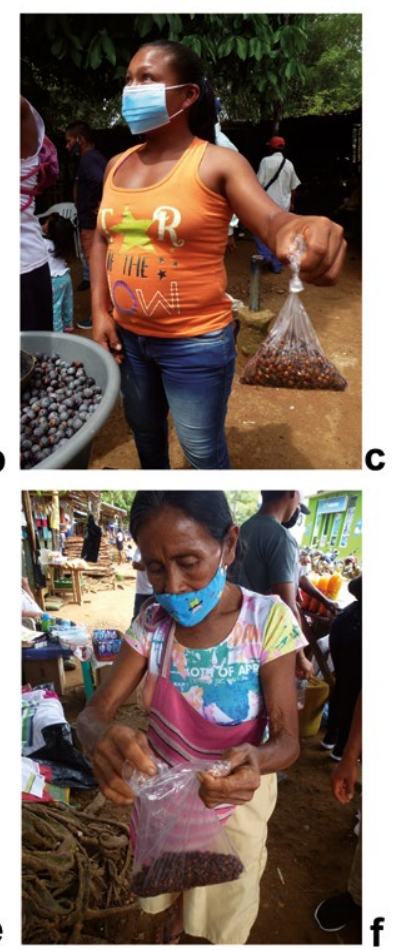

Figura 14. Comercio de manivara en el puerto de San Victorino. a, b, c. Comerciantes ofreciendo manivara para la venta. d, e, f. Proceso de empaque de manivara para la venta.

Factores que afectan la comercialización. - La comercialización de los insectos comestibles en la plaza de mercado de San Victorino de Mitú es frecuente, y por lo general, siempre hay oferta del producto. Sin embargo, en algunos meses del año no es posible vender determinado insecto, ya que está relacionado con su época de abundancia (calendario ecológico), lo que sería uno de los factores por los cuales la oferta del producto disminuye.
De acuerdo con los Cubeo, Siriana y Tatuya, otros factores que podrían influir en la comercialización son las mismas actividades humanas. Para el caso de los mojojoy, en ocasiones no se encuentran suficientes palmas caídas para recolectar larvas o se caen en los pantanos en cuyo caso no es posible encontrar el mojojoy. En contraste, cuando se abren chagras, muchas veces los nidos y termiteros de hormigas arriera y manivara se ven afectados. Así mismo, se menciona que el uso excesivo de 
"venenos" (insecticidas) para controlar las plagas de los cultivos podría estar afectando las mismas poblaciones de hormiga arriera. Factores biológicos también podrían estar relacionados, como el mismo comportamiento de las hormigas y termitas que pueden abandonar un nido para colonizar otras áreas, o la presencia de enemigos naturales como el oso hormiguero. Algunos Cubeo mencionan que las fuertes lluvias puede ser un factor que afecte la abundancia de termitas comestibles.

\section{Discusión}

Culturalmente, y dentro de la etnobiología, la palabra insecto puede llegar a ser tan compleja que no existe una definición para la cual sea posible referirse al grupo de organismo reales que lo conforman, sin excluir alguna implicación conceptual (Aparicio-Aparicio et al. 2018). Las comunidades indígenas del oriente amazónico no utilizan el término insecto en la vida diaria. Los entrevistados identificaron como insectos a hormigas, abejas y avispas (Hymenotera), moscas y zancudos (Diptera), cucarrones (Coleoptera), comejenes (Blattodea: Isoptera), mariposas (Lepidoptera), grillos (Orthoptera), o chicharras (Hemiptera). En todos los casos, las respuestas obtenidas concordaron con especímenes pertenecientes al grupo de los insectos sensu stricto. En este grupo etnotaxonómico también se mencionaron otros tipos de artrópodos como arañas (Aranae) y ciempiés (Myriapoda). En muy pocas ocasiones, también se mencionaron caracoles de tierra (Molusca) y lombrices de tierra (Oligochaeta).

Debido a la capacidad que tienen los insectos para desplazarse, en especial aquellos que pueden volar, regularmente son clasificados en agrupaciones asociadas a su medio natural o hábitat, por lo que pueden situarse en etnocategorías pertenecientes al aire (cielo), agua y tierra. De acuerdo con Aparicio-Aparicio et al. (2018), este aspecto podría estar influenciando la adopción de la definición popular de la palabra insecto.

El consumo de larvas y pupas de avispas ha sido reportado en otros grupos indígenas de la Amazonía colombiana. Los Tukano colectan nidos de avispas sociales de especies como Polybia rejecta (Fabricius), Apoica thoracica Buysson y Agelaia angulata (Fabricius), siendo frecuentemente incluidas en la alimentación diaria de la población, y proporcionando una contribución significante al consumo dietario proteínico en determinados momentos del año (Dufour 1987). Las etnias Bará y Tuyuca, localizadas al suroriente del municipio de Mitú, en el departamento del Vaupés, consumen larvas de avispas sociales del género Polistes Latreille (Osorno-Muñoz et al. 2014). Por otra parte, los indígenas Yukpa de la región caribe, en la frontera con Venezuela (Sierra de Perijá, Serranía de Valledupar y Serranía de los Motilones), se alimentan de varias especies de avispas Polistes Latreille y Polybia Lepeletier (Ruddle, 1975). Sotomayor-Tribín et al. (1998) mencionan la recolección de insectos para consumo, principalmente larvas de mojojoy y de avispas, como una actividad importante del pueblo nómada de los Nukak.

La manivara es un insecto comestible ampliamente conocido en la región amazónica. Su consumo habitual, al igual que en el oriente amazónico de la región del Vaupés, se hace tostando los especímenes soldados adultos. Hasta el momento, incluyendo a la especie $S$. spinosus, se han reportado siete especies de termitas comestibles pertenecientes a los géneros Labiotermes Fontes, Macrotermes Holmgren y Syntermes Holmgren (Termitidae), las cuales se recolectan estacionalmente y son usadas como alimento por las etnias Tukano, Bará y Tuyuca (Dufour 1987, Paoletti et al. 2000, Paoletti \& Dufour 2005; Osorno-Muñoz et al. 2014, Mitsuhashi 2017). Las hormigas arrieras también constituyen un alimento común en las comunidades indígenas de la Amazonía colombiana. Soldados y hembras aladas de Atta cephalotes (L.), A. sexdens (L.) y A. laevigata (Smith), son fácilmente colectadas y consumidas tostadas o ahumadas por diferentes grupos étnicos (Posey 1978, Dufour 1987, Osorno-Muñoz et al. 2014). En la región caribe, los indígenas Yukpa también incluyen en su alimentación diferentes especies de Atta Fabricius (Ruddle 1973, Costa-Neto \& Ramos-Elorduy 2006).

El uso de orugas de polillas como alimento también ha sido estudiado en varios pueblos indígenas de la Amazonía, donde se han reportado cerca de nueve especies comestibles. Se destacan especies pertenecientes a las familias Noctuidae y Saturniidae (Ruddle 1973, Dufour 1987, Paoletti \& Dufour 2005, Osorno-Muñoz et al. 2014). El tapurú Lusura chera (Drury) (Notodontidae) constituye un nuevo registro de oruga comestible para la amazonia colombiana (Fig. 5a). El consumo de mariposas y polillas ha sido ampliamente documentado en el mundo. Las larvas sirven como alimento para muchos grupos étnicos, y pueden ser preparadas a la parrilla, en agua salada, fritas o mezcladas con otros alimentos, aportando una gran cantidad de proteínas y energía a la dieta de los pueblos indígenas (Ramos-Elorduy 1998, Ramos-Elorduy et al. 2011). Se conocen cerca de 380 especies comestibles, de las cuales la gran mayoría se consumen en estado larval, aunque de algunas especies se consumen las crisálidas y hasta los adultos (Mitsuhashi 2017). Solo en México se han identificado 67 especies comestibles de Lepidoptera, usadas como alimento por más de 25 grupos étnicos ubicados en 17 estados mexicanos (Ramos-Elorduy et al. 2011).

Uno de los insectos comestibles más conocidos en la región amazónica son las larvas del escarabajo picudo o mojojoy. Los primeros casos documentados de consumo fueron descritos por Dufour (1987) por parte de los indígenas Tukano en el Vaupés, en donde también se registró el consumo de larvas de otras especies de Coleoptera. Las larvas de mojojoy también se encuentran incluidas en la dieta de las comunidades indígenas de Bella Vista y Puerto Loro, en zona del río Tiquié de la región del Vaupés (Osorno et al., 2014), muy cercanas al área de estudio de la presente investigación.

Debido a que el mojojoy es considerado una fuente de proteínas para los indígenas amazónicos, constituyendo un alimento apetecible para las comunidades, Cerda et al. (1999) plantearon un sistema no intensivo de cría en pequeña escala, para ser desarrollado por las comunidades indígenas en la amazonia venezolana. Así 
mismo, realizaron una composición nutricional de macro y micronutrientes de la larva del mojojoy y de tres palmeras hospederas. Cristancho-Sánchez y BarragánFonseca (2011), realizaron un completo estudio sobre el uso y aprovechamiento de la larva del mojojoy en diferentes comunidades del municipio de Leticia en la amazonia colombiana. A partir de un análisis de los factores biológicos, uso y conocimiento tradicional, se presenta una propuesta de aprovechamiento del mojojoy como una alternativa de producción de origen animal para el beneficio de la población. Una de estas alternativas es el uso del mojojoy como remedio tradicional o medicamento mediante el consumo directo de las larvas o de la extracción de su aceite el cual se puede consumir con miel (Cristancho-Sánchez \& Barragán-Fonseca 2011).

De acuerdo con Costa-Neto y Ramos-Elorduy (2006), los gorgojos del género Rhynchophorus probablemente son los insectos comestibles no domesticados más ampliamente cultivados en Asia, África y Latinoamérica, debido a su importancia como fuente de alimento y al efecto positivo que generan en la economía doméstica cuando se destinan a la comercialización. En Ecuador, el consumo de los chontacuros (mojojoy) representa una herencia cultural histórica de los indígenas amazónicos (Sancho et al. 2015). De manera similar a la recolecta de mojojoy realizadas por habitantes de las comunidades del oriente amazónico, los nativos Kwichuas realizan prácticas tradicionales derribando las palmeras de chonta (Bactris gasipaes Kunth) y de morete (Mauritia flexuosa L.) que crecen en áreas pantanosas del bosque con el objetivo de promover el ataque y posterior ovoposición del chontacuro en los troncos de las palmas. Transcurridas algunas semanas, los Kwichuas regresan a cosechar las larvas bien desarrolladas de estos gorgojos (Onore 1997). Los indígenas Jotï del Amazonas venezolano, utilizan sus conocimientos tradicionales para manejar la relación natural de los insectos con plantas hospederas como Oenocarpus bacaba Mart. con el fin de obtener las larvas de Rhynchophorus palmarum (L.) y Rhinostomus barbirostris (Fabricius) favoreciendo su producción y permitiéndoles de manera artificial la cosecha de insectos comestibles (Choo et al. 2009). Este tipo de iniciativas pueden ser planteadas en las comunidades estudiadas aledañas al municipio de Mitú, como parte de un programa de rescate de los valores culturales donde se analicen aspectos relacionados con el aprovechamiento del recurso biológico y la seguridad alimentaria.

Otra de las especies de insectos frecuentemente consumidas en la zona del Tiquié son las hormigas (Atta spp.) y termitas, comejenes o manivara (Syntermes spp.), especies que se cosechan estacionalmente. Estos insectos comestibles presentan las mayores frecuencias de consumo durante los meses de abril, durante el vuelo nupcial de las hormigas arrieras y en octubre en el vuelo de las termitas. De acuerdo con Osorno et al. (2014), en abril, después del incremento de la precipitación, disminuyen la pesca y la fauna de cacería y se incrementa el consumo de insectos. Esto coincide con el calendario ecológico preliminar para estos insectos, de acuerdo con la información suministrada por los participantes de las diferentes etnias en el presente estudio (Tabla 5). Sin embargo, los Cubeo mencionan que la manivara puede llegar a ser más frecuente en abril y septiembre.

A pesar de que el consumo de insectos no forma parte de la alimentación diaria, es importante resaltar cómo este recurso se convierte en un elemento complementario y casi que primordial al momento de la preparación de diferentes alimentos. Así mismo se evidencia un esmero en la preparación, en donde se tienen en cuenta cuidados y tiempo de cocción con el fin de obtener un alimento nutritivo. De esta manera, es importante incentivar el consumo de otro tipo de fuente de alimento como son los insectos, preservando y manteniendo los conocimientos tradicionales y uso de insectos comestibles en las diferentes preparaciones que acompañan alimentos diarios como el casabe, el pescado, el caldo de quiñapira, entre otros. Nuevas formas de preparación y combinación de alimentos en la dieta diaria, incluyendo insectos consumibles, son una alternativa para implementar en el mantenimiento de una seguridad alimentaria en las comunidades del oriente amazónico. Esta apropiación tradicional está relacionada y se puede evidenciar en las formas de comercialización de insectos comestibles, en donde los comerciantes de mojojoy, manivara, hormiga arriera y tapurú se preocupan por ofrecer productos frescos y bien presentados, que atraigan el interés de los compradores y así garantizar la venta del producto.

A pesar de que la oferta de insectos comestibles en el mercado de San Victorino de Mitú depende de la estacionalidad de algunas especies, la comercialización es frecuente a lo largo de cada año. Esto representa una actividad económica significativa para los vendedores y los habitantes locales que dependen también de la venta de otros productos como pescado, casabe, plátano y ají, y que tienen en el comercio de insectos comestibles una fuente de ingresos alternativa, teniendo en cuenta la realidad de una economía de subsistencia. El consumo de insectos puede convertirse en una solución para la seguridad alimentaria de los países en desarrollo, en donde el aumento del consumo puede aumentar la comercialización, siendo necesario diseñar regulaciones para la cría, producción, distribución y comercialización de insectos comestibles (Pino-Moreno \& Reyes-Prado 2020). Las prácticas de comercialización en otras regiones del mundo, donde existe una cultura arraigada de entomofagia como México, Australia y algunos países de África, han demostrado que los insectos representan un recurso prometedor para la economía de las comunidades rurales (Mariod 2020, Pino-Moreno \& Reyes-Prado 2020, Ponce-Reyes \& Lessard 2021).

Las especies de insectos comestibles consumidas por las comunidades del oriente amazónico pueden constituirse en un recurso renovable, planteando estrategias de aprovechamiento sostenible de sus poblaciones, teniendo en cuenta aspectos como la preservación de la biodiversidad ecológica y ecosistémica de la región amazónica del Vaupés. De acuerdo con los factores de comercialización analizados, las especies de insectos comestibles en el oriente amazónico presentan un potencial comer- 
cial y condiciones favorables para establecer sistemas de aprovechamiento, en donde es necesario incluir factores biológicos como las asociaciones planta-huésped de los estadios larvales y su adaptación al medio. Por lo consiguiente, se sugiere plantear una serie de parámetros básicos para el aprovechamiento de insectos comestibles, que incluya el estudio de factores como:

a) la densidad de la población: en donde se pueda establecer una estimación de cuantos individuos pueden ser aprovechables sin detrimento poblacional;

b) estacionalidad: referente a una estimación de la cantidad de producto en una determinada época del año garantizando la renovabilidad del recurso;

c) impacto de cosecha: desarrollando prácticas amigables y formas adecuadas de obtener el recurso sin afectar su capacidad de regeneración;

d) posibilidades de manejo: identificando estrategias para el seguimiento y monitoreo del recurso in situ, con el fin de optimizar la producción y mantenimiento del recurso y

e) impacto ambiental: estimando las implicaciones ecológicas que pueden generar el aprovechamiento del recurso.

Para promover y mantener el uso de insectos como recursos alimenticios sin afectar el hábitat natural, se requieren programas prácticos de cría en cautiverio y se necesitan ensayos a pequeña y gran escala para desarrollarse en las comunidades. El diseño de mini sistemas productivos que puedan sostener a los pobladores es una forma de reducir el impacto sobre los bosques y áreas naturales, proporcionando herramientas innovadoras para las comunidades locales y constituyéndose en un potencial para posibles actividades ecoturísticas (Paoletti et al. 2000, Baiano 2020, Guiné et al. 2021, Selaledi et al. 2021).

Los resultados obtenidos en el presente estudio representan información preliminar para el establecimiento $y$ fortalecimiento de investigaciones asociadas al uso y producción de insectos con fines alimenticios en las comunidades indígenas del oriente amazónico. Mediante el fomento de actividades y prácticas comunitarias, que se enfoquen en educación y capacitación, los pobladores de las diferentes comunidades pueden ser conscientes de los beneficios o perjuicios que las actividades de extracción, aprovechamiento o producción generan sobre las dinámicas ecológicas de las especies comestibles. De igual manera, este primer acercamiento hacia el conocimiento y uso de insectos como fuente de alimento, permitirá proponer alternativas culturalmente viables en comunidades específicas de la región (e.g. Santa María de Itapinima, Piracemo, Santa Elena del Tiposo, Ceima Cachivera, Mituseño-Urania), para el establecimiento de estrategias de uso y aprovechamiento direccionadas a mejorar la seguridad alimentaria de los habitantes, manteniendo y respetando los conocimientos y saberes ancestrales de las comunidades.

\section{Literatura citada}

Aparicio-Aparicio JC, Costa-Neto E, Paulino de Araújo G. 2018. Etnotaxonomía mixteca de algunos insectos en el municipio de San Miguel El Grande, Oaxaca, México. Revista Etnobiología 16(2): 58-75.

Arango-Gutiérrez GP. 2011. Breve reseña histórica de la entomofagía en Colombia. In: E.M. Costa-Neto, org. Antropoentomofagia: Insetos na alimentação humana. UEFS Editora. Feria de Santana. Pp. 171-199.

Baiano A. 2020. Edible insects: An overview on nutritional characteristics, safety, farming, production technologies, regulatory framework, and socio-economic and ethical implications. Trends in Food Science \& Technology 100: 35-50. https://doi.org/10.1016/j. tifs.2020.03.040

Banjo AD, Lawal OA, Songonuga EA. 2006. The nutritional value of fourteen species of edible insects in Southwestern Nigeria. African Journal of Biotechnology 5(3): 298301.

Becker, V0. 2014. Checklist of the new world Notodontidae (Lepidoptera: Noctuoidea). Lepidoptera Novae 7(1): $1-40$.

Bernard T, Womeni HM. 2017. Entomophagy: Insects as food. In: V. Shields (eds.). Insect Physiology and Ecology. Rijeka InTech. London.

Cabrera G, Nates-Parra G. 1999. Uso de las abejas por comunidades indígenas: Los Nukak y las abejas sin aguijón. Encuentro IUSSI Bolivariana Memorias. Universidad Nacional de Colombia y Fondo FEN Colombia. Bogotá.

Cárdenas D, Arias JC, Vanegas J, Jiménez D, Vargas O, Gómez L. 2007. Plantas útiles y promisorias en la Comunidad de Wacuraba (Caño Cuduyarí) en el departamento de Vaupés (Amazonía colombiana). Instituto Amazónico de Investigaciones Científicas Sinchi. Bogotá.

Cerda H, Martínez R, Briceño N, Pizzoferrato L, Hermoso D, Paoletti M. 1999. Cría, análisis nutricional y sensorial del picudo del cocotero Rhynchophorus palmarum (Coleoptera: Curculionidae), insecto de la dieta tradicional indígena amazónica. Ecotrópicos 12(1): 25-32. http://www.saber.ula.ve/handle/123456789/25491

Choo J. Zent E, Simpson BB. 2009. The Importance of Traditional Ecological Knowledge for Palm-weevil Cultivation in the Venezuelan Amazon. Journal of Ethnobiology 29(1): 113-128. https://doi.org/10.2993/0278-077129.1.113

Constantino, R. 1995. Revision of the neotropical termite genus Syntermes Holmgren (Isoptera: Termitidae). The university of Kansas Bulletin 55(13): 455-518.

Corporación C.D.A. 2013. Plan de Integral de vida Cubeo zona UDIC. CDA. Mitú. 145 pp.

Costa-Neto EM. 2003. Insetos como fontes de alimentos para o homem: valoração de recursos considerados repugnantes. Interciencia 28 (3): 136-140.

Costa-Neto E, Ramos-Elorduy J. 2006. Los insectos comestibles de Brasil: etnicidad, diversidad e importancia en la alimentación. Boletín Sociedad Entomológica Aragonesa (38): 423-442.

Costa-Neto EM, Santos-Fita F, Serrano-González R. 2012 La investigación etnoentomológica y la conservación de la biodiversidad. Boletín de la Sociedad Entomológica Aragonesa 51: 367-369.

Cristancho-Sánchez SV, Barragán-Fonseca KB. 2011. Análisis del Sistema de aprovechamiento del gusano mojojoy (Rynchophorus palmarum) (Coleoptera: Curculio- 
nidae) en el municipio de Leticia-Amazonas, Colombia. In: R. Monroy-Martínez, A. García-Flores, J. M. Pino-Moreno, R. Monroy-Ortiz, eds. Etnozoología. Un enfoque binacional México-Colombia. Universidad Autónoma del Estado de Morelos. Centro de Investigaciones Biológicas. Cuernavaca. Pp. 131-199.

DeFoliart GR. 2005 Overview of role of edible insects in preserving biodiversity. In: M. G. Paoletti, ed. Ecological Implications of Minilivestock: Potential of Insects, Rodents, Frogs and Snails. Science Publishers Inc. Enfield. Pp. 123-140.

Dufour DL. 1987. Insects as food: a case study from Northern Amazon. American Anthropologist 89: 383-397. https://doi.org/10.1525/aa.1987.89.2.02a00070

Fernández F, Castro-Huertas V, Serna F. 2015. Hormigas Cortadoras de hojas en Colombia: Acromyrmex \& Atta. Universidad Nacional de Colombia. Colombia.

Gahukar RT. 2011. Entomophagy and human food security. International Journal of Tropical Insect Science 31(3): 129-144. https://doi.org/10.1017/ S1742758411000257

Gasca-Álvarez HJ. 2005. El significado de los escarabajos (Coleoptera: Scarabaeoidea) en una comunidad Uitoto de Leticia, Amazonas (Colombia): Una exploración preliminar a su conocimiento etnoentomológico. Boletín Sociedad Entomológica Aragonesa 36: 309-315.

Guiné RPF, Correia P, Coelho C, Costa CA. 2021. The role of edible insects to mitigate challenges for sustainability. Open Agriculture 6: 24-36. https://doi.org/10.1515/ opag-2020-0206

Halloran A, Flore R, Vantomme P, Roos N. 2018. Edible Insects in Sustainable Food Systems. Springer. Switzerland. https://doi.org/10.1007/978-3-319-74011-9

Jara F. 1996. La miel y el aguijón, taxonomía zoológica y etnobiología como elementos en la definición de las nociones de género entre los Andoke (Amazonía Colombiana). Journal de la Societé des Americanistes 82: 209-258. https://doi.org/10.3406/jsa.1996.1637

Johnson DV. 2010 The contribution of edible forest insects to human nutrition and to forest management: current status and future potential, In: D.B. Durst, D.V. Johnson, R.N. Leslie, K. Shono, eds. Forest Insects as Food: Humans Bite Back. Proceedings of a Workshop on Asia-Pacific Resources and Their Potential for Development. FAO Regional Office for Asia and the Pacific. Bangkok. Pp. 5-22.

Jongema Y. 2017. List of edible insects of the world. Wageningen University \& Research, Wageningen, the Netherlands. Available at: http://tinyurl.com/mestm6p.

Kim TK, Yong HI. Kim YB, Kim HW, Choi YS. 2019. Edible insects as a protein source: a review of public perception, processing technology, and research trends. Food Science of Animal Resources 39: 521-540. https://doi. org/10.5851/kosfa.2019.e53

Mariod AA. 2020. African edible insects as alternative source of food, oil, protein and bioactive components. Springer. Switzerland.

Mitsuhashi J., 2017. Edible Insects of the World. CRC Press. Boca Raton. https://doi.org/10.1201/9781315367927

Molina J. 2015. Si fueran de comer no serían tantos. Hipótesis 18: 31-41.

Mozhui L, Kakati LN, Changkija S. 2017. A study on the use of insects as food in seven tribal communities in $\mathrm{Na}$ galand, Northeast India. Journal of Human Ecology
60(1): 42-54. https://doi.org/10.1080/09709274.2 017.1399632

Onore G. 1997. A brief note on edible insects in Ecuador. Ecology of Food and Nutrition 36(2-4): 277-285. https:// doi.org/10.1080/03670244.1997.9991520

Osorno-Muñoz M, Atuesta-Dimian N, Jaramillo-Hurtado LF, Sua-Tunjano S., Barona-Colmenares A, RoncancioDuque N. 2014. La despensa de Tiquié. Diagnóstico y manejo comunitario de la fauna de consumo en la Guayana colombiana. Instituto Amazónico de Investigaciones Científicas - Sinchi. Ministerio de Ambiente y Desarrollo Sostenible. Bogotá.

Paoletti MG, Buscardo E, Dufour DL. 2000. Edible invertebrates among Amazonian Indians, a disappearing knowledge. Environment, Development and Sustainability, 2: 195225. https://doi.org/10.1023/A:1011461907591

Paoletti MG, Dufour DL. 2005. Edible invertebrates among Amazonian Indians: A critical review of disappearing knowledge. In: M.G. Paoletti, ed. Ecological Implications of Minilivestock, Potential of Insects, Rodents, Frogs and Snails. Science Publishers Inc. Enfield. Pp. 293-342

Pino-Moreno JM, Ganguly A. 2016. Determination of fatty acid content in some edible insects of Mexico. Journal of Insects as Food and Feed 2(1): 37-42. https://doi. org/10.3920/JIFF2015.0078

Pino-Moreno J.M, Reyes-Prado H. 2021. Commerce of Edible Insects in the State of Morelos, Mexico. Journal of Insect Science 20(5): 1-7. https://doi.org/10.1093/jisesa/ ieaa106

Phillips OL, Gentry AH, Reynel C, Wilkin P, Galvez-Durand B. 1994. Quantitative ethnobotany and Amazonian conservation. Conservation Biology 8: 225-248. https:// doi.org/10.1046/j.1523-1739.1994.08010225.x

Ponce-Reyes R, Lessard BD. 2021. Edible Insects - A roadmap for the strategic growth of an emerging Australian industry. CSIRO. Canberra.

Posey DA. 1978. Ethnoentomological survey of Amerind groups in lowland Latin America. Florida Entomologist 61: 225-229. https://doi.org/10.2307/3494211

Ramos-Elorduy J. 1998. Creepy crawly cuisine. Park Street (Inner Traditions International). Rochester.

Ramos-Elorduy J. 2009. Anthropo-entomophagy: cultures, evolution and sustainability. Entomological Research, 39: 271-288. https://doi.org/10.1111/j.17485967.2009.00238.x

Ramos-Elorduy J, Pino-Moreno JM. 2004. Los Coleoptera comestibles de México. Anales del Instituto de Biología, Universidad Nacional Autónoma de México, Serie Zoología 75(1): 149-183.

Ramos-Elorduy J, Pino-Moreno JM, Vázquez AI, Landero I, Oliva-Rivera H, Camacho V. 2011. Edible Lepidoptera in Mexico: Geographic distribution, ethnicity, economic and nutritional importance for rural people. Journal of Ethnobiology and Ethnomedicine 7(2): 1-22. https:// doi.org/10.1186/1746-4269-7-2

Reyes-Mora A. 2011. Insectos utilizados como alimento y su relación con la mitología en la comunidad Murui de San José del Encanto, Amazon, Colombia. In: E.M. Costa-Neto, org. Antropoentomofagia: Insetos na alimentação humana. UEFS Editora, Feria de Santana. Pp. 201-222.

Ruddle K. 1973. The Human Use of Insects: Examples from the Yukpa. Biotrópica 5(2): 94-101. https://doi. org/10.2307/2989658 
Sancho D, Álvarez-Gil M, Fernández-Sánchez L. 2015. Insectos y alimentación. Larvas de Rhynchophorus palmarum L, un alimento de los pobladores de la Amazonía Ecuatoriana. Entomotrópoica 30 (14): 136-149.

Selaledi L, Hassan Z, Manyelo TG, Mabelebele M. 2021. Insects' production, consumption, policy, and sustainability: what have we learned from the indigenous knowledge systems? Insects 12(432): 1-18. https://doi. org/10.3390/insects12050432

Seni A, 2017. Edible insects: future prospects for dietary regimen. International Journal of Current Microbiology and Applied Sciences, 6(8): 1302-1314. https://doi. org/10.20546/ijcmas.2017.608.158

Sotomayor-Tribín HA, Mahecha-Rubio D, Franky-Calvo CE, Cabrera-Becerra G, Torres-Leguízamo ML. 1998. La nutrición de los Nukak, una sociedad Amazónica en proceso de contacto. Maguare 13: 199-142.

Skotnicka M, Karwowska K, Kłobukowski F, Borkowska A, Pieszko M. 2021. Possibilities of the Development of Edible Insect-Based Foods in Europe. Foods 10: 766. https://doi.org/10.3390/foods10040766

Tobolkova B. 2019. Edible insects-the future of a healthy diet? Novel Techniques in Nutrition and Food Science 4(2): 326-328. https://doi.org/10.31031/ NTNF.2019.04.000584

van Huis A. 2013. Potential of Insects as Food and Feed in Assuring Food Security. Annual Review of Entomology, 58:563-83. https://doi.org/10.1146/annurev-ento-120811-153704

van Huis A, Van Itterbeeck J, Klunder H, Mertens E, Halloran A, Muir G, Vantomme P., 2013. Edible insects: future prospects for food and feed security. FAO Forestry Paper no. 171. Food and Agriculture Organization of the United Nations (FAO). Rome.

Xiaoming C, Ying F, Hong Z. 2010. Review of the nutrition value of edible insects. In: D. B. Durst, D. V. Johnson, R. N. Leslie, K. Shono, eds. Forest Insects as Food: Humans Bite Back. Proceedings of a Workshop on Asia-Pacific Resources and Their Potential for Development. FAO Regional Office for Asia and the Pacific. Bangkok. Pp. 85-92.

Yen AL. 2010. Edible insects and other invertebrates in Australia: future prospects. In: D. B. Durst, D. V. Johnson, R. N. Leslie, K. Shono, eds. Forest Insects as Food: Humans Bite Back. Proceedings of a Workshop on Asia-Pacific Resources and Their Potential for Development. FAO Regional Office for Asia and the Pacific. Bangkok. Pp. 65-83.

\section{Agradecimientos / Acknowledgments:}

Para la realización de las encuestas hechas por William González, coautor del manuscrito, se ha contado con el apoyo logístico del Instituto Amazónico de Investigaciones Científicas- SINCHI (sede Mitú) gracias a la colaboración y gestiones de Luis Fernando Jaramillo, coordinador de sede y de Mariela Osorno, directora del programa de fauna. Un agradecimiento especial a Maribel Palacios Bocanegra, auxiliar de investigación de la subsede Mitú, por su colaboración en la organización, escaneo y envío de los formatos de entrevistas diligenciados. Agradecemos a Dairon Cárdenas por la determinación taxonómica del Turi. De igual manera, agradecemos la colaboración de Rudolf Scheffrahn, Fernando Fernández, Giovanni Fagua, Liliana Prada, Vitor Becker, Thibaud Decaens, Ryan St Laurent y Carlos Sarmiento por la confirmación y determinación taxonómica de las especies de insectos comestibles. Agradecemos también a dos revisores anónimos por sus valiosos comentarios y sugerencias que nos ayudaron a mejorar el manuscrito. La presente investigación forma parte del proyecto "Diversidad de insectos como fuente de alimento para las comunidades indígenas del Oriente Amazónico", desarrollado por el Instituto SINCHI y apoyado por el Programa de Estancias Postdoctorales del Ministerio de Ciencia, Tecnología e Innovación - MINCIENCIAS (Convocatoria 848 de 2019).

Conflicto de intereses / Competing interests:

Los autores no incurren en conflictos de intereses.

\section{Rol de los autores / Authors Roles:}

H.J.G.A y W.R: Conceptualización, análisis formal, investigación; H.J.G.A: metodología; redacción versión borrador, redacción, revisión y edición. Todos los autores han leído y están de acuerdo con la versión publicada de este manuscrito.

\section{Fuentes de financiamiento / Funding:}

La presente investigación forma parte del proyecto "Diversidad de insectos como fuente de alimento para las comunidades indígenas del Oriente Amazónico", desarrollado por el Instituto SINCHI y apoyado y financiado por el Programa de Estancias Postdoctorales del Ministerio de Ciencia, Tecnología e Innovación- MINCIENCIAS (Convocatoria 848 de 2019).

Aspectos éticos / legales; Ethics / legals:

Los autores declaran que no violaron ni omitieron normas éticas o legales en esta investigación. 
Anexo 1. Formulario de entrevistas $A$

\title{
DIVERSIDAD DE INSECTOS COMO FUENTE DE ALIMENTO PARA LAS COMUNIDADES INDÍGENAS DEL ORIENTE AMAZÓNICO
}

\author{
Encuesta semiestructurada de tipo sincrónico \\ (Una misma pregunta hecha a individuos diferentes) \\ FORMATO A \\ Percepción de los insectos por parte de los habitantes
}

Fecha: Entrevista No.

Lugar (Municipio, Vereda, Sitio, etc)

Nombre: Sexo: Edad:

Oficio (si aplica)

1. ¿Qué entiendes por la palabra INSECTO?

2. ¿Qué tipo de insectos conoces en el lugar (pueblo, vereda, comunidad) dónde vives? ¿Cuáles son sus nombres comunes?

3. ¿Cuáles son los lugares más comunes en donde puedes encontrar insectos?

4. ¿Consideras a los insectos animales dañinos, perjudiciales o peligrosos? ¿O, por lo contrario, consideras que son beneficiosos, inofensivos, buenos?

5. ¿Conoces algún insecto en el lugar (pueblo, vereda, comunidad) dónde vives que sea utilizado como fuente de alimento, comestible? 
Anexo 2. Formulario de entrevista B

\title{
DIVERSIDAD DE INSECTOS COMO FUENTE DE ALIMENTO PARA LAS COMUNIDADES INDÍGENAS DEL ORIENTE AMAZÓNICO
}

\author{
Encuesta semiestructurada de tipo sincrónico \\ (Una misma pregunta hecha a individuos diferentes) \\ FORMATO B \\ Información sobre insectos usados como alimento
}

Fecha: Entrevista No.

Lugar (Municipio, Vereda, Sitio, etc)

Nombre:

Sexo: Edad:

Oficio (si aplica)

1. ¿Utiliza algún tipo de insecto (bicho) como fuente de alimento?

2. ¿Dónde y cómo recolecta (captura) esos insectos? ¿Utiliza alguna técnica específica?

3. ¿A qué hora del día y en qué época del año recolecta (captura) los insectos para consumo?

4. ¿Cuánto tiempo emplea para recolectar (capturar) los insectos empleados para consumo?

5. ¿Cómo prepara el alimento empleando insectos? ¿Existe una forma tradicional de preparación? ¿Tiempo de cocción? ¿Acompañamientos?

6. ¿El consumo de insectos hace parte de su alimentación diaria?

7. ¿Cuáles son los insectos más comunes empleados como alimento en el lugar donde vive (pueblo, vereda, comunidad)? 
Anexo 3. Formulario de entrevistas $C$.

\title{
DIVERSIDAD DE INSECTOS COMO FUENTE DE ALIMENTO PARA LAS COMUNIDADES INDÍGENAS DEL ORIENTE AMAZÓNICO
}

\author{
Encuesta semiestructurada de tipo sincrónico \\ (Una misma pregunta hecha a individuos diferentes)
}

\section{FORMATO C}

Información sobre comercialización de insectos comestibles

Fecha: Entrevista No.

Lugar (Municipio, Vereda, Sitio, etc)

Nombre:

Sexo: Edad:

Oficio (si aplica)

1. ¿Cuál(es) es(son) el(los) insecto(s) comestible que usted vende?

2. ¿Cómo lo comercializa? ¿Dónde lo vende? ¿Con que frecuencia? ¿Quién le compra el producto?

3. ¿De dónde viene el producto y como lo obtuvo?

4. ¿Cuál es el precio de compra/venta del producto?

5. ¿Cómo debe ser la calidad del producto para que pueda ser comercializado? ¿Qué condiciones debe tener para que se venda?

6. ¿Qué factores pueden afectar la comercialización del producto? 
Anexo 4. Lista de comunidades indígenas de los entrevistados que participaron en la investigación.

\begin{tabular}{|c|c|c|c|}
\hline 1 & 12 de octubre & 22 & Puente Lata - Vía Santa Cruz \\
\hline 2 & Acaricuara - Mitú Vaupés & 23 & Puerto Asis - Caño Aviju \\
\hline 3 & Arara - Río Cuduyari & 24 & Puerto Colombia - Río Vaupés \\
\hline 4 & Balarmino - Mitu & 25 & Puerto Corroncho - Río Vaupés \\
\hline 5 & Camuti - Rio Cuduyari & 26 & Puerto Gavilán - Río Caduyari \\
\hline 6 & Ceima Cachivera & 27 & Puerto Golondrina \\
\hline 7 & Cerrito Verde & 28 & Puerto Inaya - Río Vaupés \\
\hline 8 & Cucura & 29 & Puerto López - Rio Cuyudari \\
\hline 9 & GarraFa - Rio Cuduyari & 30 & Puerto Pato - Rio Cuduyari \\
\hline 10 & Guamal -Río Vaupés & 31 & Puerto Pinilla - río Vaupés \\
\hline 11 & La Libertad - Ozimí Mitú & 32 & Puerto San Victorino de Mitú \\
\hline 12 & Macaquiño & 33 & Puerto Pupuña - Río Vaupés \\
\hline 13 & Mitú & 34 & Santa María de Itapinima - Rio Cuduyari \\
\hline 14 & Mitu, barrio Cuervo Araoz & 35 & Santa Elena de Tiposo - Rio Cuduyari \\
\hline 15 & Mituseño Urania & 36 & Santa Rita - Querarí \\
\hline 16 & Murutinga & 37 & Santa Rosalia - Cañoyi \\
\hline 17 & Pacuativa - Rio Cuduyari & 38 & Timbo de Betania \\
\hline 18 & Patio Bonito Río Vaupés & 39 & Tierra Grata - Río Vaués \\
\hline 19 & Pira - Rio Cuduyari & 40 & Tucunaré - Río Vaupés \\
\hline 20 & Piracemo - Río Cuyudari & 41 & Valencia Cano Río Vaupés \\
\hline 21 & Pueblo Nuevo & 42 & Wasay - Rio Vaupés médio \\
\hline
\end{tabular}

